\title{
基于细节增强和平行特征刺激脉冲耦合神经网络的 医学图像融合算法
}

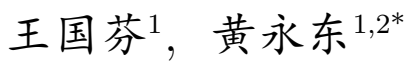 \\ 1. 北方民族大学图像处理与理解研究所, 银川 750021 \\ 2. 大连民族大学理学院, 大连 116600 \\ *通信作者. E-mail: huang_yongdong@163.com
}

收稿日期: 2018-11-01；修回日期：2019-01-06；接受日期: 2019-03-19; 网络出版日期: 2020-02-12

国家自然科学基金项目 (批准号: 11761001, 11761003) 和宁夏科技创新领军人才项目 (批准号: KJT2016002) 资助

\begin{abstract}
摘要医学图像融合的目的是将多幅多模态医学图像的信息整合到一幅图像上, 此图像有助于临床 诊断, 帮助医生精确观察细微病变, 缩短病人的治疗周期. 本文提出了一种新的解剖图像和功能图像的 融合算法，选取了局部拉普拉斯滤波 (local Laplacian filtering, LLF) 作为融合过程的分解工具, 该工 具在增强细节的同时保护边缘, 保证解剖图像的细节信息不被功能图像的颜色信息遮盖. 首先, 利用 LLF 将原图像分解为近似图和一系列细节图. 其次, 对于近似图, 结合区域能量和边缘能量提出一个 改进的局部能量取大的融合规则; 对于细节图, 采用参数自适应的简化脉冲耦合神经网络 (parameteradaptive simplified pulse coupled-neural network, PA-SPCNN) 模型进行细节图融合, 选取改进的拉普 拉斯和 (novel sum-modified-Laplacian, NSML) 与彩色显著特征信息 (color saliency feature, CSF) 分别 作为解剖图像和功能图像所对应的 PA-SPCNN 模型的外部刺激输入。最后, 使用逆 LLF 变换获得融 合图像. 仿真实验表明本文所提算法在主观评价和客观评估方面与已有算法相比具有一定优势.
\end{abstract}

关键词图像处理, 图像融合, 医学图像处理, 局部拉普拉斯滤波, 脉冲耦合神经网络, 拉普拉斯和, 彩 色显著特征, 边缘能量

\section{1 引言}

多模态医学图像融合是指按特定的分解工具和融合规则将来自于多模态的多幅图像信息融合成 一幅图像,该图像集成了多幅图像的特征,信息更丰富,视觉效果更好. 医学图像大致被分为两类: 解 剖图像和功能图像. 解剖图像包括计算机断层扫描 (computed tomography, CT) 图像和核磁共振成像 (magnetic resonance imaging, MRI) 图像, 该类图像有较高的空间分辨率, 主要提供解剖结构信息. 功

\footnotetext{
引用格式: 王国芬, 黄永东. 基于细节增强和平行特征刺激脉冲耦合神经网络的医学图像融合算法. 中国科学: 信息科学, 2020, 50: 239-260, doi: 10.1360/N112018-00295

Wang G F, Huang Y D. Medical-image-fusion algorithm based on a detail-enhanced and pulse-coupled neural-network model stimulated by parallel features (in Chinese). Sci Sin Inform, 2020, 50: 239-260, doi: 10.1360/N112018-00295
} 
能图像包括正电子发射断层摄影 (positron emission tomography, PET) 图像和单光子发射计算机断层 (single photon emission computed tomography, SPECT) 图像, 这些图像空间分辨率较低, 但是可以清 晰地显示人体组织或器官的代谢状态和血流状态. 解剖图像和功能图像的融合有助于检测疾病区域以 及定位病变位置. 融合图像被广泛地用于处理由人体、器官或细胞反映的医学问题, 也可以帮助医生在 诊断过程中给出综合有效的治疗方案, 缩短病人的治疗周期 [1]. 本文聚焦 MRI-PET 和 MRI-SPECT 医学图像融合.

多模态医学图像融合过程如下: 首先利用多尺度分解工具将多幅原图像分解到不同尺度上得到一 系列蕴含不同特征不同信息的子带，再根据子带的特征信息分别设计合适的融合规则进行子带融合， 最后利用融合子带进行重构得到融合图像. 分解工具的选取关系着原图像信息的充分提取与完美展现.

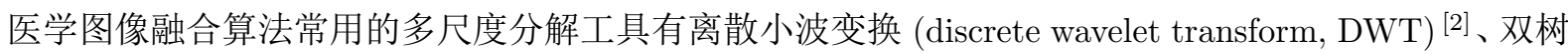
复小波变换 (dual-tree complex wavelet transform, DTCWT) ${ }^{[3]}$ 、拉普拉斯金字塔 (Laplacian pyramid, LP) ${ }^{[4]}$ 、梯度金字塔 (gradient pyramids, GP) ${ }^{[5]}$, 以及拥有平移不变性、各向异性的非下采样轮廓波 变换 (nonsubsampled contourlet transform, NSCT) ${ }^{[6]}$ 和非下采样剪切波变换 (nonsubsampled shearlet transform, NSST) ${ }^{[7]}$ 等. 此外, 学者们还将边缘保护滤波作为多尺度分解工具, 此类分解工具有较强

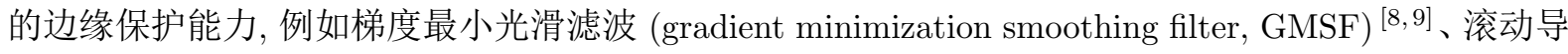
向滤波 (rolling guidance filter, RGF) ${ }^{[10]}$ 等. 局部拉普拉斯滤波 (local Laplacian filtering, LLF) ${ }^{[11]}$ 也 是边缘保护滤波, 它克服了传统拉普拉斯金字塔滤波在融合医学图像过程中会引入退化的边缘和光晕 效应的缺陷. 在特定参数下, LLF 还能够在保护图像边缘信息的同时增强图像的细节信息. 另一方面, 融合规则的设计直接影响着融合结果, 近年来提出了大量的经典多模态医学图像融合规则, 并取得了 较好的成果. 例如, 利用导向滤波更新权重的融合规则 [12], 基于结构张量和共轭梯度优化相结合的融 合规则 ${ }^{[13]}$, 基于平行特征融合的融合规则 ${ }^{[14,15]}$, 基于感兴趣信息 (information of interest, IOI) 的融 合规则 ${ }^{[16]}$, 基于主成分分析 (principal component analysis, PCA) 的融合规则 ${ }^{[17]}$, 基于鲁棒主成分分 析 (robust principal component analysis, RPCA) 的融合规则 ${ }^{[18]}$, 基于稀疏表示 (sparse representation, SR) 的融合规则 ${ }^{[19]}$, 以及基于改进空间频率的稀疏表示 (modified sparse representation, MSR) 融合规 则 [20]. 由于脉冲耦合神经网络 (pulse coupled-neural network, PCNN) 是基于动物视觉感知建立的模 型, 对图像特征的刻画非常符合人类视觉感知, 因此在图像融合领域基于 PCNN 模型的融合规则也备 受学者关注, 如空间频率作外部刺激的脉冲耦合神经网络 (spatial frequency-motivated pulse coupled neural networks, SF-PCNN) 的融合规则 ${ }^{221]}$, 基于自适应链接权重 PCNN 模型的融合规则 ${ }^{[22]}$, 基于 脉冲发放皮层模型 (spiking cortical model, SCM) 的融合规则 ${ }^{[23]}$, 以及基于参数自适应的简化脉冲耦 合神经网络 (parameter-adaptive simplified pulse coupled-neural network, PA-SPCNN) 的融合规则 [24]. 上述模型中, 要么参数是常数, 导致不具有自适应性, 要么参数较多增加了计算复杂性, 要么具有自适 应性但其捕捉信息形式过于单一. 众所周知, 优秀的医学图像融合结果应该既能很好地综合两幅原图 像的信息, 还能够使得各自的信息都能充分地在融合图像中被呈现. 由于本文聚焦于研究解剖图像和 功能图像的融合, 融合这两类图像应当考虑到两类图像有不同的信息呈现能力和表现方式, 比如灰度 解剖图像主要提供解剖结构信息, 彩色功能图像主要提供代谢和血流的颜色深浅信息, 因此融合过程 中需要选取能充分提取解剖图像的结构信息和功能图像的颜色信息的工具, 此外还应考虑到功能图像 的彩色信息不应该在融合图像中覆盖掉解剖图像的结构信息.

LLF 已在文献 [16] 中被用于图像分解与重构, 该工具既能达到增强细节的目的, 又能避免在融 合过程中可能会出现功能图像的颜色信息覆盖掉解剖图像细节信息的现象. 因此, 本文选取 LLF 作 为图像分解和重构工具. 同时, 选取改进的拉普拉斯和 (novel sum-modified-Laplacian, NSML) [25] 与 
彩色显著特征信息 (color saliency feature, CSF) ${ }^{[26]}$ 分别作为解剖图像和功能图像在 PA-SPCNN 模 型中的外部刺激输入, 其中 NSML 方法从八个方向充分提取图像的结构信息, 且 CSF 方法根据颜色 刺激提取彩色图像的显著性特征, 也就是说这两种信息提取方式能够充分提取解剖图像的结构信息和 体现功能图像的颜色显著信息, 改善了 PA-SPCNN 模型中外部刺激输入单一的缺陷. 此外, 由于近似 图主要包含的是能量信息和基本结构信息, 因此, 由局部能量和边缘能量构成的改进的局部能量取大 (improved-local energy maximum, I-LEM) 融合规则被用于近似图的融合, 弥补了近似图融合过程只利 用局部能量取大方法的考虑不足. 本文所提算法的步骤大致如下: 利用 LLF 将原图像分解为一个近 似图和一系列细节图, PA-SPCNN 作为细节图融合模型, NSML 和 CSF 分别作为解剖图像和功能图 像对应模型的外部刺激输入, 局部能量和边缘能量构成的改进的局部能量取大融合规则被用于近似图 的融合, 最后将逆 LLF 操作应用到融合后的近似图与细节图中得到融合结果图像. 本文有如下创新 点：(1) 对于 LLF 分解后得到的近似图, 根据近似图的内容信息, 引入了边缘能量的概念并结合区域 能量提出一个改进的局部能量取大的融合规则. 即对于任一个像素, 利用该像素点所对应的边缘能量 和区域能量来综合选取该像素点的加权系数. (2) 对于 LLF 分解后得到的细节图, 参数自适应的简化 脉冲耦合神经网络模型被用于细节图融合, 本文首次考虑到解剖图像和功能图像有不同的呈现特征方 式, 故选取改进的拉普拉斯和与彩色显著特征分别作为解剖图像和功能图像所对应的 PA-SPCNN 模 型的外部刺激输入, 以达到充分提取解剖图像的结构信息和功能图像的颜色信息的目的.

本文的其余部分安排如下, 第 2 节是理论简介, 第 3 节是所提融合算法, 第 4 节是参数讨论及主 客观评价, 第 5 节是总结.

\section{2 理论简介}

\section{1 局部拉普拉斯滤波}

2011 年, Paris 等提出了局部拉普拉斯滤波: LLF 处理图像时, 首先是对原图像 $I$ 进行高斯 (Gauss) 函数滤波操作 $\left(G_{i+1}=\operatorname{downsample}\left(G_{i}\right), G_{0}=I\right)$ 得到一系列图像层 $G_{i}(i=0, \ldots, L)$, 所有图像层的 集合称为高斯金字塔, 其中 $G_{i+1}$ 的分辨率是 $G_{i}$ 的一半. 第 $i$ 层拉普拉斯金字塔 $S_{i}$ 可由高斯金字塔 的第 $i$ 层与第 $i+1$ 层上采样后相减得到 $\left(S_{i}=G_{i}-\operatorname{upsample}\left(G_{i+1}\right)\right.$, 且拉普拉斯金字塔的最顶层被 定义为 $S_{L}=G_{L}$ ). 其次是通过点向单调重映射函数 (point-wise monotonic remapping) 更新获得一个 新的中间图像 $I^{\prime}$,

$$
I^{\prime}(v)= \begin{cases}g+\operatorname{sign}(v-g) \sigma_{r}\left(|v-g| / \sigma_{r}\right)^{\alpha}, & \text { if }|v-g| \leqslant \sigma_{r}, \\ g+\operatorname{sign}(v-g)\left(\beta\left(|v-g|-\sigma_{r}\right)+\sigma_{r}\right), & \text { otherwise, }\end{cases}
$$

其中 $i$ 表示金字塔的层数, $v=(x, y, i)$ 是位于第 $i$ 层 $(x, y)$ 位置的像素值, sign 表示符号函数, $g$ 是高 斯金字塔作用于原图像产生的值.

随后, LLF 对中间图像 $I^{\prime}$ 计算其拉普拉斯金字塔系数 $S_{i}\left[I^{\prime}\right]$ 并将此系数赋为输出图像 $O$ 的拉普 拉斯金字塔系数, 再通过折叠 (collapse) 所有金字塔层得到滤波输出 $O$,

$$
O=\operatorname{Collapse}\left(S_{i}\left[I^{\prime}\right]\right)
$$

其中, 折叠过程即为重构图像过程 $\left(G_{i}=S_{i}+\operatorname{upsample}\left(G_{i+1}\right),(i=L-1, \ldots, 0)\right)$, 即由 $G_{L}\left(S_{L}\right), S_{L-1}$, $S_{L-2}, \ldots, S_{0}$ 得到 $G_{L-1}, G_{L-2}, \ldots, G_{0}$ 的过程, 最后滤波输出 $O=G_{0}$, 滤波输出 $O$ 与输入图像 $I$ 大 小一致. 
LLF 的 3 个参数分别是强度参数 $\sigma_{r}$ 、细节参数 $\alpha$ 和边缘参数 $\beta$. 强度参数 $\sigma_{r}$ 作为阈值用于区 分细节信息与边缘信息. 当 $|v-g| \leqslant \sigma_{r}$ 时, $v$ 应当被处理为细节信息, 反之, $v$ 就应该被处理为边缘信 息. 细节参数 $\alpha$ 与细节信息密切相关. 边缘参数 $\beta$ 对边缘信息来说至关重要. 当 $\alpha>1$ 且 $\beta=1$ 时, 对 输入图像进行 LLF 操作具有模糊细节的效果. 当 $0<\alpha<1$ 且 $\beta=1$ 时, 对输入图像进行 LLF 操作 是一个增强细节的过程. 当 $\alpha=1$ 且 $0 \leqslant \beta<1$ 时, 对输入图像进行 LLF 操作是一个边缘感知色调映 射的过程. 当 $\alpha=1$ 且 $\beta>1$ 时, 对输入图像进行 LLF 操作相当于对输入图像进行逆色调映射操作. 当 $0<\alpha<1$ 且 $0 \leqslant \beta<1$ 时, 对输入图像进行 LLF 操作相当于对输入图像同时进行细节增强和色 调映射操作. 当 $\alpha=1$ 且 $\beta=1$ 时, 输入图像的系数不会改变. 对于一个彩色的输入图像, LLF 输出是 通过分别对彩色图像的 3 个颜色通道滤波得到. 为了对融合图像进行保护强边缘的同时增强细节, 本 文选取 $\alpha=0.25, \beta=1, \sigma_{r}=0.4$, 参数选取思想方法来源于文献 [16]. 图 1 展示了输入的彩色图像和 灰度图像经过 LLF 滤波前后的对比图. 从图中可以清楚地看到, 原彩色图像 (图 1(a1)) 经过 LLF 后 得到的彩色图像 (图 1(a2)) 的细节明显增强了许多, 尤其是花瓣的茎向纹理部分 (红色三角形标注的 区域), 叶子的轮廓部分 (红色粗圆标注的区域) 以及背景土地 (红色方框标注的区域) 中模糊的痕迹都 被明显地增强. 图 1(b1), (b2), (b3) 分别是灰度图像 (MRI), 灰度图像执行 LLF 滤波前的像素分布图, 灰度图像执行 LLF 滤波后的像素分布图. 从图中可以观察到, 在执行 LLF 滤波前灰度图像 (MRI 图 像) 的像素分布较光滑, 在经过 LLF 后 MRI 图像的像素分布明显要尖锐了一些. 也就是说, 执行 LLF 后的 MRI 图像的细节比原图像细节更多、更清晰, 图中标注的地方可以看到明显差异.

\section{2 彩色显著特征}

CSF 方法是基于颜色刺激的, 也就是说, 彩色图像的颜色鲜明区域应当赋予大的显著性值; 相反, 均质区域应当赋予小的显著性值. CSF 旨在检测显著性特征, 该特征既包含突出的对象, 也包含传达 上下文的背景. CSF 的测量由颜色、方向和强度等低层次显著性特征驱动, 它遵循 4 个原则: (1) 考虑 局部低层次特征, 如对比度和颜色信息; (2) 考虑全局特征, 抑制频繁发生的特征的同时保持偏离规范 的特征; (3) 符合人类视觉组织规律; (4) 显著性区域易于中心化. 计算输入图像的 CSF 包括局部全局 单尺寸显著性、多尺寸显著性和中心优先 3 步操作, 具体过程如下:

首先, 像素 $p$ 在 $r$ 尺寸下的单尺寸显著性 $s_{p}^{r}$ :

$$
s_{p}^{r}=1-\exp \left\{-\frac{1}{K} \sum_{k=1}^{K} d\left(q_{p}^{r}, q_{k}^{r}\right)\right\},
$$

其中 $K$ 表示与尺寸为 $r \times r$ 的图像块 $q_{p}^{r}$ 最相似的图像块 $q_{k}^{r}(k=1,2, \ldots, K)$ 的总个数, 一般取 $K=64$. 其中 $d\left(q_{p}^{r}, q_{k}^{r}\right)$ 定义如下:

$$
d\left(q_{p}^{r}, q_{k}^{r}\right)=\frac{d_{\text {color }}\left(q_{p}^{r}, q_{k}^{r}\right)}{1+c \cdot d_{\text {position }}\left(q_{p}^{r}, q_{k}^{r}\right)},
$$

其中 $d_{\text {color }}\left(q_{p}^{r}, q_{k}^{r}\right)$ 表示在 Lab 空间中图像块 $q_{p}^{r}, q_{k}^{r}$ 之间的欧氏距离, 通常取 $c=3, d_{\text {position }}\left(q_{p}^{r}, q_{k}^{r}\right)$ 表 示图像块 $q_{p}^{r}$ 与 $q_{k}^{r}$ 之间的位置欧氏距离.

其次, 多尺寸显著性通过降低背景像素的显著性来改善显著性和非显著性区域之间的一致性. 由 于每个尺寸下都能计算出由关注焦点组成的最受关注的区域 (通常 $s_{p}^{r}>0.8$ 就认为该像素点是关注 焦点), 且与关注焦点相近的区域比远离关注焦点的区域更有探索价值, 故由如下表达式计算像素 $p$ 的 显著性:

$$
\hat{f}_{p}=\frac{1}{M} \sum_{r \in R} s_{p}^{r}\left(1-d_{\mathrm{foci}}^{r}(p)\right),
$$




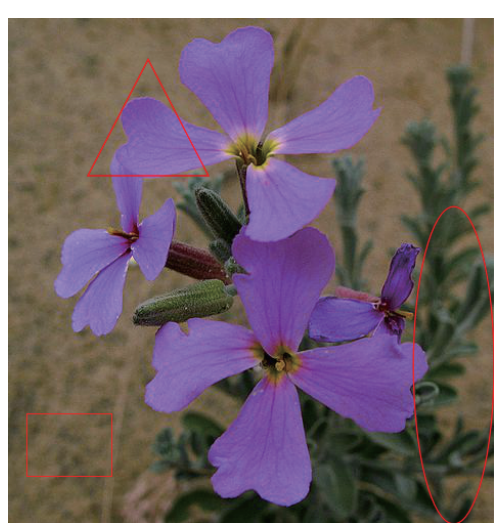

(a1)

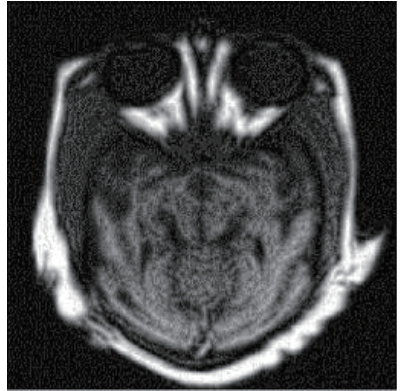

(b1)

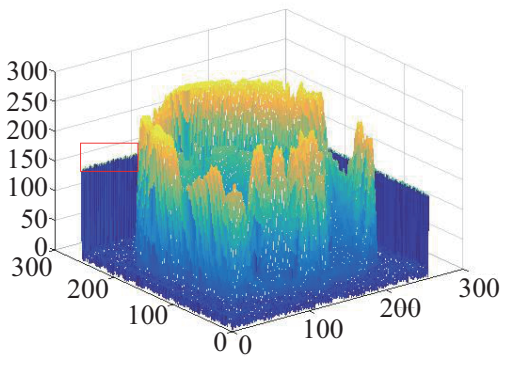

(b2)

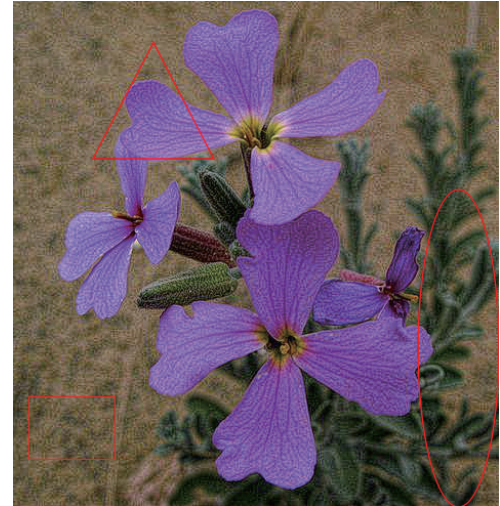

(a2)

图 1 (网络版彩图) LLF 滤波应用于彩色图与灰度图前后的对比图. (a1) 彩色图; (a2) 滤波后的图; (b1) 灰度图; (b2) 滤波前灰度图的像素分布图; (b3) 滤波后灰度图的像素分布图

Figure 1 (Color online) Comparison of results before and after applying LLF to color and gray images. (a1) Color image; (a2) color image after LLF; (b1) gray image; (b2) pixel distribution map of gray image before LLF; (b3) pixel distribution map of gray image after LLF

其中 $R=\left\{r_{1}, \ldots, r_{M}\right\}$ 表示像素块尺寸 $r$ 的 $M$ 个不同值, $d_{\mathrm{foci}}^{r}(p)$ 表示在 $r$ 尺寸上像素 $p$ 与距离它最 近的关注焦点之间的位置欧氏距离.

最后, 图像 $I$ 的 CSF 由下式获得:

$$
\operatorname{CSF}(I)=\hat{f}_{p} G_{p},
$$

其中 $G_{p}$ 是位于图像中心的二维高斯函数 $G_{p}(u, v)=\frac{1}{2 \pi \sigma^{2}} \mathrm{e}^{-\left(u^{2}+v^{2}\right) /\left(2 \sigma^{2}\right)}$, 其中 $\sigma$ 是正态分布的标 准差.

图 2 是 PET 医学图像和 SPECT 医学图像进行彩色显著特性提取的例子. 从图中观察到, 显著 图里面着重强调了有高对比度的红色和蓝色部分, 越亮的地方显著亮度值也就越高. 也就是说 CSF 完 全保留了 PET 和 SPECT 的颜色亮度信息, 也就是疾病的高亮度信息.

\section{3 梯度域引导滤波}

梯度域引导滤波 (gradient domain guided image filtering, GDGF) ${ }^{[27]}$ 在引导滤波 (guided image filtering, GF) ${ }^{[28]}$ 的基础上引入了一个显式一阶边缘感知约束, 使得它保护边缘的能力比引导滤波更 


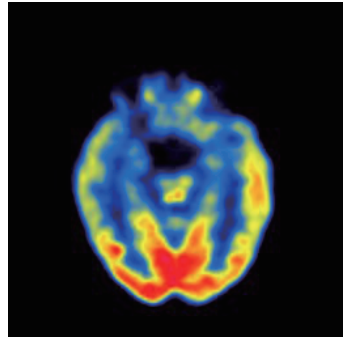

(a1)

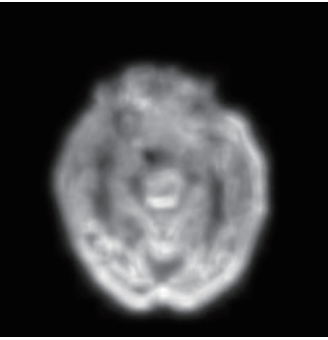

(a2)

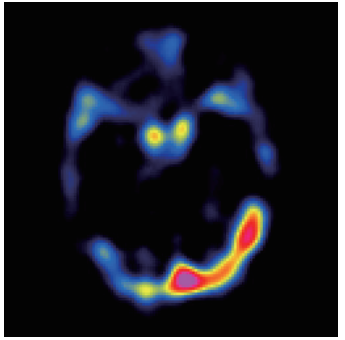

(b1)

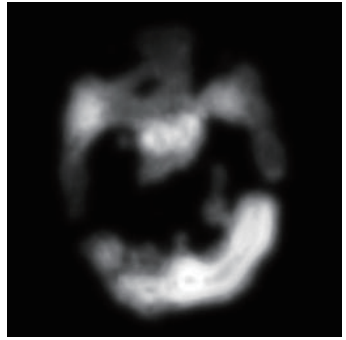

(b2)

图 2 (网络版彩图) 彩色显著特征的提取. (a1) PET 图像; (a2) PET 图像的 CSF; (b1) SPECT 图像; (b2) SPECT 图像的 CSF

Figure 2 (Color online) Color saliency feature extraction. (a1) PET image; (a2) CSF of PET image; (b1) SPECT image; (b2) CSF of SPECT image

好. GDGF 有如下成本函数:

$$
E\left(a_{k}, b_{k}\right)=\sum_{i \in \Omega_{r}(k)}\left[\left(a_{k} I_{i}+b_{k}-P_{i}\right)^{2}+\frac{\epsilon}{\Gamma_{I, k}}\left(a_{k}-\gamma_{k}\right)\right],
$$

其中 $I$ 是引导图像, $P$ 是输入图像, $\Omega_{r}(k)$ 表示以像素 $k$ 为中心, $r$ 为半径的方形窗口, $\gamma_{k}$ 是一个边缘 感知滤波因子, $\Gamma_{I, k}$ 是一个边缘感知滤波权重因子, 它衡量像素 $k$ 在整个引导图像中的重要性, $\gamma_{k}$ 和 $\Gamma_{I, k}$ 表示如下:

$$
\begin{gathered}
\gamma_{k}=1-\frac{1}{1+e^{\varphi_{k}}}, \quad \varphi_{k}=\frac{4\left(S_{k}-\mu_{\boldsymbol{S}}\right)}{\mu_{\boldsymbol{S}}-\min (\boldsymbol{S})}, \\
\Gamma_{I, k}=\frac{1}{N} \sum_{i=1}^{N} \frac{S_{k}+\varepsilon}{S_{i}+\varepsilon},
\end{gathered}
$$

其中 $N$ 表示引导图像的像素总个数, $\varepsilon$ 是一个很小的正数, 通常设置为 $10^{-6} \times D^{2}, D$ 是输入图像的 动态变化范围. $S_{k}$ 被定义为 $\sigma_{I, 1}(k) \cdot \sigma_{I, r}(k), \sigma_{I, 1}(k)$ 和 $\sigma_{I, r}(k)$ 是引导图像 $I$ 分别在窗口 $\Gamma_{1}$ 和 $\Gamma_{r}$ 中 的标准差, 通常设置 $r=16 . \boldsymbol{S}=\left\{S_{1}, \ldots, S_{N}\right\}, \mu_{\boldsymbol{S}}$ 表示所有 $S_{i}(i=1, \ldots, N)$ 的均值.

$a_{k}$ 和 $b_{k}$ 的最优值计算如下:

$$
\begin{gathered}
a_{k}=\frac{\mu_{I \odot P, r}(k)-\mu_{I, r}(k) \mu_{P, r}(k)+\frac{\epsilon}{\Gamma_{I, k}} \gamma_{k}}{\sigma_{I, r}^{2}(k)+\frac{\epsilon}{\Gamma_{I, k}}}, \\
b_{k}=\mu_{P, r}(k)-a_{k} \mu_{I, r}(k),
\end{gathered}
$$

其中 $\odot$ 表示点积, $\mu_{I \odot P, r}(k), \mu_{I, r}(k), \mu_{P, r}(k)$ 分别是 $I \odot P, I, P$ 在窗口 $\Omega_{r}(k)$ 中的均值.

最后的输出 $q_{i}$ 由下式给出:

$$
q_{i}=\mu_{a(i)} I_{i}+\mu_{b(i)},
$$

其中, $\mu_{a(i)}$ 和 $\mu_{b(i)}$ 表示在所有窗口之中的 $a_{k}$ 和 $b_{k}$ 的均值, 分别计算如下:

$$
\mu_{a(i)}=\frac{1}{\left|\Omega_{r}(i)\right|} \sum_{k \in \Omega_{r}(i)} a(k), \quad \mu_{b(i)}=\frac{1}{\left|\Omega_{r}(i)\right|} \sum_{k \in \Omega_{r}(i)} b(k),
$$

$\left|\Omega_{r}(i)\right|$ 表示 $\Omega_{r}(i)$ 的基数. 


\section{4 参数自适应的简化脉冲耦合神经网络模型}

传统的神经网络模型中, 如何确定参数依旧是最大的挑战, 例如链接强度、各种振幅以及衰减系 数. 为了克服参数难以选取的不足, Chen 等 ${ }^{[29]}$ 提出了一种参数自适应的简化脉冲耦合神经网络模型. 该模型如下:

$$
\begin{gathered}
F_{i j}[n]=S_{i j}, \\
L_{i j}[n]=V_{L} \sum_{k l} W_{i j k l} Y_{k l}[n-1], \\
U_{i j}[n]=\mathrm{e}^{-\alpha_{f}} U_{i j}[n-1]+F_{i j}[n]\left(1+\beta L_{i j}[n]\right), \\
Y_{i j}[n]= \begin{cases}1, & \text { if } U_{i j}[n]>E_{i j}[n-1], \\
0, & \text { otherwise, }\end{cases} \\
E_{i j}[n]=\mathrm{e}^{-\alpha_{e}} E_{i j}[n-1]+V_{E} Y_{i j}[n] .
\end{gathered}
$$

上述模型中, $F_{i j}[n]$ 和 $L_{i j}[n]$ 分别表示位于 $(i, j)$ 的像素在第 $n$ 次迭代过程中的外部刺激输入与 链接输入. $V_{L}$ 是链接输入 $L_{i j}[n]$ 的振幅, 它通过突触权重 $W_{i j k l}$ 与前一点火状态 $Y_{k l}[n-1]$ 一起控制 $L_{i j}[n]$, 其中突触权重矩阵

$$
W_{i j k l}=\left[\begin{array}{ccc}
0.5 & 1 & 0.5 \\
1 & 0 & 1 \\
0.5 & 1 & 0.5
\end{array}\right] .
$$

内部活性 $U_{i j}[n]$ 由两部分构成: 前一部分是前一状态的内部活性的指数衰减部分; 后一部分是 $L_{i j}[n]$ 和 $F_{i j}[n]$ 通过链接权重 $\beta$ 组合的一部分. $Y_{i j}[n]$ 依赖于内部活性 $U_{i j}[n]$ 和前一状态的动态阈值 $E_{i j}[n-1]$, 当 $U_{i j}[n]$ 大于 $E_{i j}[n-1]$ 时有 $Y_{i j}[n]=1$, 意味着点火; 反之, 当 $Y_{i j}[n]=0$, 意味着不点火. $E_{i j}[n]$ 表示第 $n$ 次迭代过程中的动态阈值, 它是通过指数衰减函数 $\mathrm{e}^{-\alpha_{e}}$ 和系数 $V_{E}$ 共同调制的. 图 3 是 PA-SPCNN 模型的结构图.

实际上, $\alpha_{f}$ 值越小, 内部活性 $U_{i j}[n]$ 的动态范围就越大, 且它与归一化图像的标准差 $\sigma$ 成逆相关 关系; 最优直方图间值 $I^{\prime}$ 与图像最大强度值 $I_{\max }$ 共同决定 $\beta$ 的取值; 此外, $\beta V_{L}$ 在整个模型中是一 个定值且 $V_{L}$ 的取值不影响实验结果, 故 $V_{L}$ 取定值; 将最大图像强度值、最优直方图阈值和点火强度 范围相结合获得 $V_{E}$ 和 $\alpha_{e}$ 的计算公式, PA-SPCNN 模型中的所有参数有如下计算公式:

$$
\begin{aligned}
& \alpha_{f}=\log \left(\frac{1}{\sigma(I)}\right), \beta=\frac{\left(I_{\max / I^{\prime}}\right)}{6 V_{L}}, \\
& V_{L}=1, \quad V_{E}=\mathrm{e}^{-\alpha_{f}}+1+6 \beta V_{L}, \\
& \alpha_{e}=\ln \frac{V_{E}}{I^{\prime}\left(\frac{1-\mathrm{e}^{-3 \alpha_{f}}}{1-\mathrm{e}^{-\alpha_{f}}}+6 \beta V_{L} \mathrm{e}^{-\alpha_{f}}\right)} .
\end{aligned}
$$

\section{3 所提算法}

由于功能图像是彩色图像, 而解剖图像是灰度图像, 故在融合过程中需要采取颜色空间转换 ${ }^{[30,31]}$. 本文所提算法首先利用 3.1 小节所提分解算法将解剖图像和功能图像分解成近似图和一系列细节图; 接着将分解后的 RGB 彩色图像 (功能图像的近似图或细节图) 转换到 YUV 空间, 再由于 YUV 颜色 


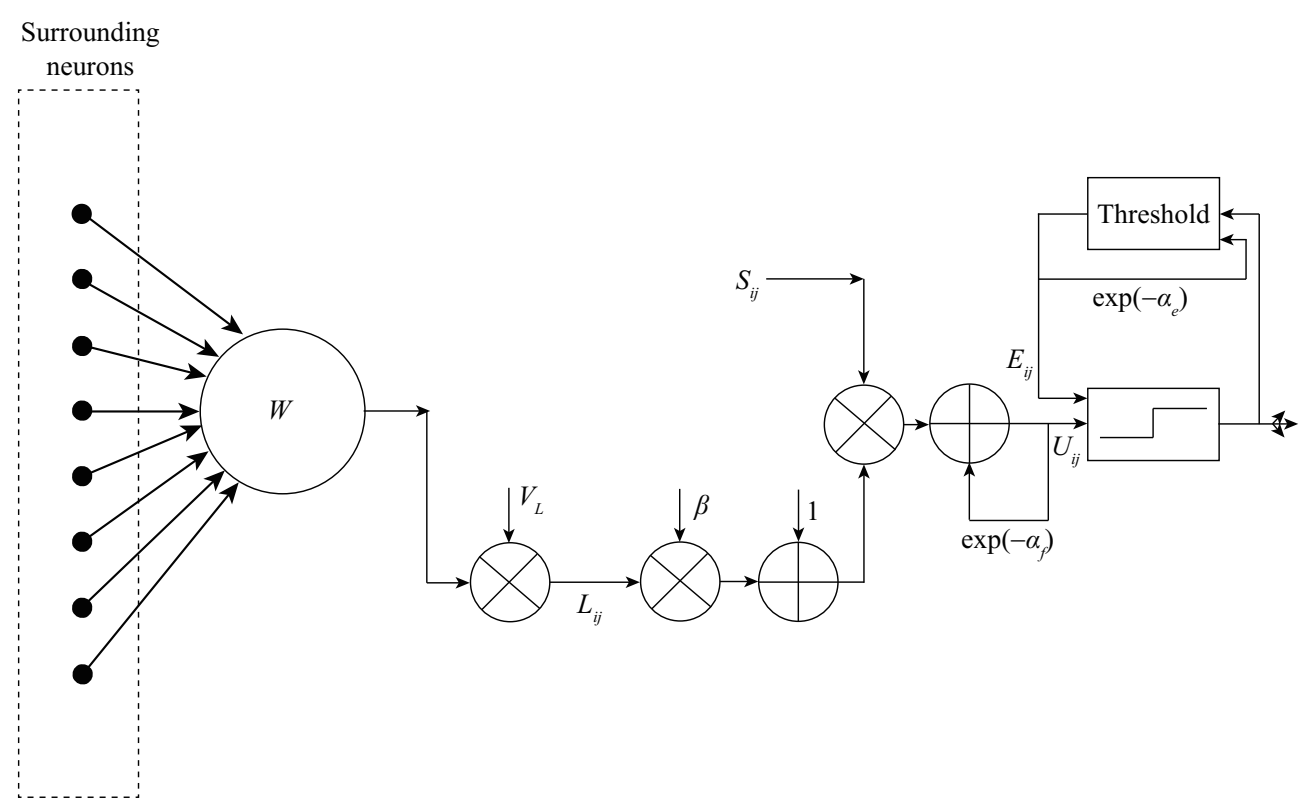

图 3 PA-SPCNN 模型的结构图

Figure 3 The diagram of PA-SPCNN model

空间中 $\mathrm{Y}$ 表示强度, 而 $\mathrm{U}$ 和 $\mathrm{V}$ 表示颜色信息, 故将 $\mathrm{Y}$ 通道图像与解剖图像按 3.2 和 3.3 小节所提的 融合规则融合得到新的 $Y$ 通道, 最后联合 UV 通道的颜色信息使用颜色空间转换得到新的 RGB 彩色 图像, 称之为融合后的近似层图像或细节层图像; 最后, 使用 3.4 小节所提方法得到融合结果. 图 4 是 本文所提融合算法的流程图 ( $L=3$ 时), 其中 RGB to YUV 表示图像从 RGB 颜色空间转到 YUV 颜 色空间, YUV to RGB 则表示图像从 YUV 颜色空间转到 RGB 颜色空间.

\section{1 图像分解}

利用 LLF 将原图像分解为一个近似图和一系列细节图. 近似图提供了原图像的低频信息, 保留 了原图像的主要能量以及基本轮廓. 细节图展示的是原图像的高频信息, 主要包含了边缘、细节和纹 理等信息. 原图像经过 LLF 分解后, 可由如下公式表示:

$$
A=\sum_{i=1}^{L-1} R_{A}^{i}+G_{A}^{L}, \quad B=\sum_{i=1}^{L-1} R_{B}^{i}+G_{B}^{L},
$$

其中 $A$ 表示解剖图像, $B$ 表示功能图像, $L$ 表示本文所提方法里 LLF 的分解层数, $R_{A}^{i}, R_{B}^{i}$ 表示两原 图像的细节层, $G_{A}^{L}, G_{B}^{L}$ 表示两原图像的近似层.

\section{2 近似图的融合规则}

由于近似图包含图像的绝大部分能量与轮廓信息. 传统的加权平均的融合规则会让融合图像产生 低对比度、信息融合不优的现象. 传统的局部能量取大方法先计算像素位置的局部能量, 融合过程中 在同一像素位置选取能量较大的像素作为融合像素. 然而, 这样的选取仅仅考虑了近似层的能量问题, 近似层还包括原图像的基本轮廓, 因此原图像的轮廓理应被考虑到融合过程中. 众所周知, 边缘保护 滤波在滤波过程中保留强边缘的同时滤掉大细节信息, 因此基于边缘保护滤波考量边缘轮廓信息是一 个合适的选择. GDGF 将一阶边缘感知约束引入到已有的图像滤波器中, 使滤波后的图像有更好的视 


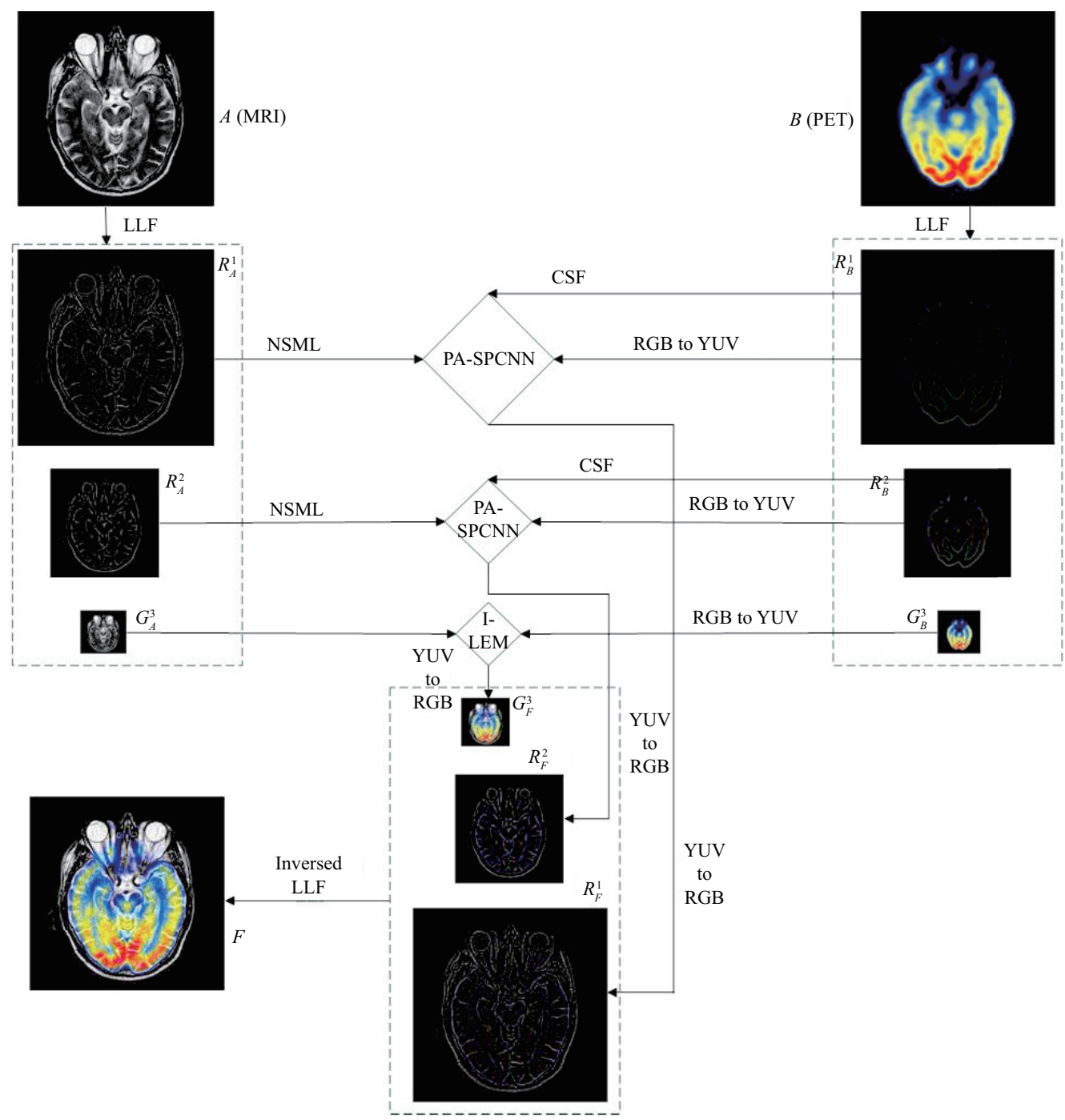

图 4 (网络版彩图) 本文所提算法流程图 $(L=3)$

Figure 4 (Color online) The flow chart of the proposed algorithm $(L=3)$

觉效果, 尤其是边缘轮廓部分. 基于以上分析, 本文提出了一种改进的局部能量取大方法. 首先计算近 似图的局部区域能量, 再利用 GDGF 得到近似图的边缘能量, 最后计算权重并得到融合的近似图, 具 体过程如下:

Step 1. 计算近似图 $G_{I}^{L}(I=A, B)$ 的局部能量 $E_{I}$ :

$$
E_{I}(x, y)=\sum_{m=1}^{w} \sum_{n=1}^{w}\left[G_{I}^{L}(x+m, y+n)\right]^{2} \times W(m, n)
$$

其中 $(x, y)$ 表示图 $G_{A}^{L}$ 和 $G_{B}^{L}$ 像素的位置, $w$ 表示局部窗口半径, 本文选取 $w=3 . W$ 是一个 $3 \times 3$ 的 
权重矩阵, 本文取

$$
W=\frac{1}{16}\left[\begin{array}{lll}
1 & 2 & 1 \\
2 & 4 & 2 \\
1 & 2 & 1
\end{array}\right] .
$$

Step 2. 使用 GDGF 处理近似图的局部能量图, 同时近似图 $G_{I}^{L}$ 作为引导图, 得到边缘能量图 $E_{I}^{\text {edge }}$ :

$$
E_{I}^{\text {edge }}=\operatorname{GDGF}\left(E_{I}, G_{I}^{L}, \epsilon\right)
$$

其中 $\epsilon$ 是 GDGF 的参数, 4.1 小节中会讨论它的选取.

Step 3. 计算近似图的二值权重:

$$
\begin{gathered}
w_{A}^{L}= \begin{cases}1, & \text { if } E_{A} \cdot E_{A}^{\text {edge }}>E_{B} \cdot E_{B}^{\text {edge }}, \\
0, & \text { otherwise }\end{cases} \\
w_{B}^{L}=1-w_{A}^{L} .
\end{gathered}
$$

Step 4. 融合的近似图 $G_{F}^{L}$ 由下式获得:

$$
G_{F}^{L}=w_{A}^{L} \times G_{A}^{L}+w_{B}^{L} \times G_{B}^{L} .
$$

\section{3 细节图的融合规则}

细节图包含的是原图的边缘、细节和纹理信息. 原 PA-SPCNN 模型选取的是像素的绝对值作为 外部刺激输入, 信息采集过于单一. 本文分别选取解剖图像的改进的拉普拉斯能量和与功能图像的彩 色显著特征作为 PA-SPCNN 的外部刺激输入, 起到充分提取解剖图像的细节信息和功能图像的颜色 亮度信息的作用. 其中, 用于解剖图像的外部刺激的 NSML 表达式如下:

$$
\begin{aligned}
& \operatorname{NSML}(x, y)=\sum_{m=1}^{w} \sum_{n=1}^{w} W \times \operatorname{SML}(x, y), \\
\operatorname{SML}(x, y)= & |I(x, y)-I(x-1, y)|+|I(x, y)-I(x+1, y)| \\
& +|I(x, y)-I(x, y-1)|+|I(x, y)-I(x, y+1)| \\
& +\frac{1}{\sqrt{2}}(|I(x, y)-I(x-1, y-1)|+|I(x, y)-I(x+1, y+1)|) \\
& +\frac{1}{\sqrt{2}}(|I(x, y)-I(x+1, y-1)|+|I(x, y)-I(x-1, y+1)|),
\end{aligned}
$$

其中 $I$ 表示解剖图像的细节图 $R_{A}^{i}(i=1, \ldots, L-1)$.

细节图的融合过程如下:

Step 1. 计算解剖图像的点火次数 $T_{A, x y}^{i}(i=1, \ldots, L-1)$, NSML 作为 PA-SPCNN 的外部刺激 输入,

$$
T_{A, x y}^{i}[n]=T_{A, x y}^{i}[n-1]+Y_{A, x y}^{i}[n] .
$$

Step 2. 计算功能图像的点火次数 $T_{B, x y}^{i}(i=1, \ldots, L-1)$, CSF 作为 PA-SPCNN 的外部刺激 输入,

$$
T_{B, x y}^{i}[n]=T_{B, x y}^{i}[n-1]+Y_{B, x y}^{i}[n] .
$$




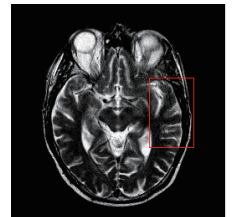

(a1)

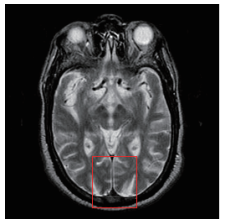

(b1)

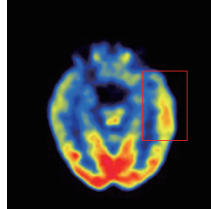

(a2)

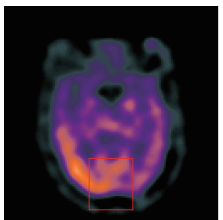

(b2)

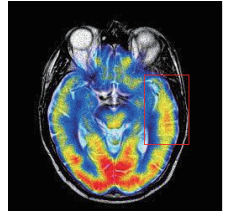

(a3)

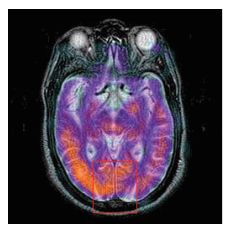

(b3)

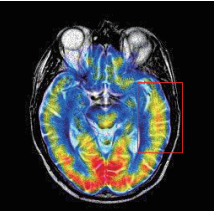

(a4)

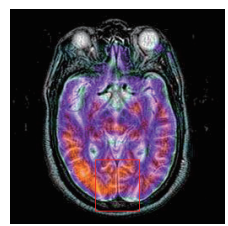

(b4)

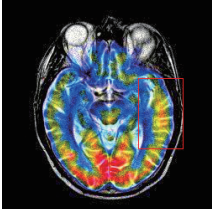

(a5)

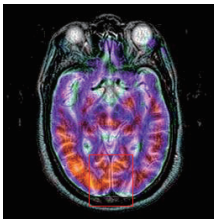

(b5)

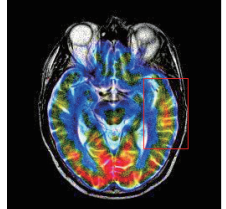

(a6)

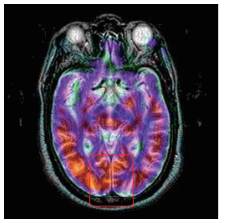

(b6)

图 5 (网络版彩图) (a1), (a2) 分别是 MRI, PET 原图像, (a3) (a6) 依次是 $L=2,3,4,5$ 的融合图像; (b1), (b2) 分别是 MRI, SPECT 原图像, (b3) (b6) 依次是 $L=2,3,4,5$ 的融合图像

Figure 5 (Color online) (a1) and (a2) are the source images of MRI and PET; (a3) (a6) are the fusion images of (a1) and (a2) when $L=2,3,4,5$, respectively; (b1) and (b2) are the source images of MRI and SPECT; (b3) (b6) are the fusion images of (b1) and (b2) when $L=2,3,4,5$, respectively

Step 3. 计算细节图的二值权重:

$$
\begin{aligned}
& w_{A}^{i}= \begin{cases}1, & \text { if } T_{A, x y}^{i}[n]>T_{B, x y}^{i}[n], \\
0, & \text { otherwise }\end{cases} \\
& w_{B}^{i}=1-w_{A}^{i} .
\end{aligned}
$$

Step 4. 融合后的细节图 $R_{F}^{i}(i=1, \ldots, L-1)$ 由下式获得:

$$
R_{F}^{i}=w_{A}^{i} \times R_{A}^{i}+w_{B}^{i} \times R_{B}^{i}
$$

\section{4 图像重构}

在得到融合后的近似图和细节图后, 通过以下重构过程得到融合图像 $F$ :

$$
F=\sum_{i=1}^{L-1} R_{F}^{i}+G_{F}^{L}
$$

\section{4 实验分析}

\section{1 参数分析}

本文所提融合算法有两个参数的取值需要进一步讨论. 一是 LLF 分解工具的分解层数, 若分解层 数过少, 信息采集不够, 融合过程信息综合不全面, 融合结果不如意; 若分解层数过多, 人工痕迹的引 入不可避免, 同样不利于融合图像的后续使用. 因此, 合适的分解层数需通过主观与客观评价标准共 同决定. 在此取分解层数分别为 $2,3,4,5$, 在 MRI-PET 与 MRI-SPECT 两组实验图像上进行实验, 其结果见图 5 和表 1 . 二是 GDGF 的模糊度参数 $\epsilon$, 由于 GDGF 用于近似图的边缘能量的获取, 它影 响着近似图的融合结果与最终的融合图像. 色调映射图像质量指标 TMQI (tone-mapped image quality index) ${ }^{[32]}$ 可用来度量参考图像和融合图像之间的亮度与对比度显著特征, 测量融合图像的结构保真 
表 1 不同层数的 LLF 分解的 SF 值

Table 1 SF values of LLF decomposition with different level $L$

\begin{tabular}{cccccc}
\hline Image pair & Metric & $L=2$ & $L=3$ & $L=4$ & $L=5$ \\
\hline MRI-PET & SF & 37.7999 & $\mathbf{3 8 . 4 2 9 7}$ & 38.3881 & 38.173 \\
MRI-SPECT & SF & 27.1511 & $\mathbf{2 8 . 4 1 8 7}$ & 28.2667 & 27.9765 \\
\hline
\end{tabular}
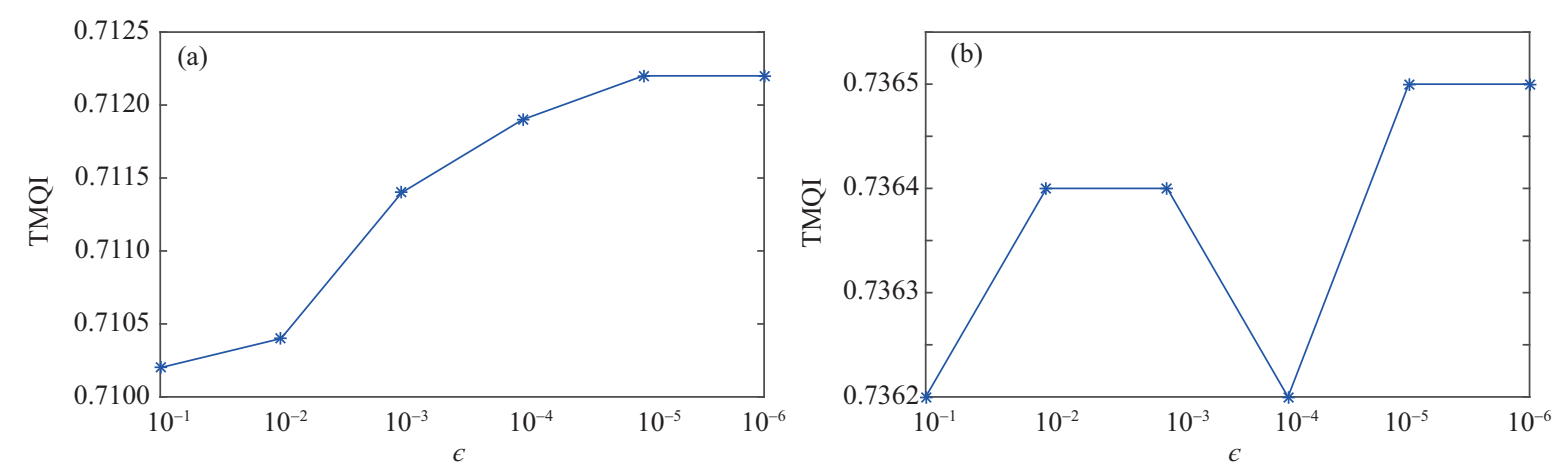

图 6 (网络版彩图) $\epsilon$ 分别取 $10^{-1} \sim 10^{-6}$ 的 TMQI 指标值的折线图

Figure 6 (Color online) TMQI values of different source image pairs when $\epsilon$ varies from $10^{-1}$ to $10^{-6}$. (a) MRI-SPECT; (b) MRI-PET

度, 指标值越大代表融合图像有更高的空间分辨率, 从输入图像中获得更多的亮度信息. 在此分别取 $10^{-6} \sim 10^{-1}$, 在 MRI-PET 与 MRI-SPECT 两组实验图像上进行实验, 融合图像的 TMQI 值分别见 图 6.

\subsection{1 确定 LLF 的分解层数 $L$}

图 5 是不同的分解层数获得的融合结果图像, 从图中可观察到分解层数越多, 融合图整体会显得 越暗沉一些, 尽管 MRI 的细节更清晰, 但是 PET 和 SPECT 的颜色信息就丢失更多. 只有图 5(a4) 和 (b4), 既能相对较好地保留 PET (SPECT) 的颜色信息又能保留 MRI 的细节信息. 也就是说当 $L=3$ 时, 融合结果的视觉效果最好. 另外, 从表 1 可以看出, 当 $L=3$ 时, 客观指标空间频率 (spatial frequency, SF) ${ }^{[33]}$ 的值最高. SF 描述的是图像在空间域的梯度分布, 度量图像的细节丰富程度, 值越 大代表图像细节越清晰, 融合效果越好. 综合客观指标和主观分析, 本文选取 $L=3$.

\subsection{2 确定 GDGF 的参数 $\epsilon$}

图 6 是 $\epsilon$ 取不同的值时, MRI-PET 与 MRI-SPECT 两组实验的融合图像所对应的 TMQI 值折线 图. 从图中可知, 当 $\epsilon=10^{-5}$ 时, 融合效果已达到最好.

综上分析, 本文选取 $L=3, \epsilon=10^{-5}$ 用于本文所有实验图像.

\section{2 所提方法的可行性分析}

为了验证本文所提的近似层融合方法和细节层融合方法的有效性, 本文分别选取了 10 组 MRIPET 和 10 组 MRI-SPECT 共 20 组医学图像用于实验, 在此部分实验中, LLF 作为所有方法的图像 分解与重构的工具. 另外, 边缘强度 (EI) ${ }^{[34]}$ 值越大代表图像边缘越锐化, 融合图像质量越好. 表 2 是 两类实验图分别使用 4 种融合方法获得的 EI 指标的平均值. 


\section{表 2 不同融合方法的 EI 平均值}

Table 2 Average EI values of different fusion methods

\begin{tabular}{cccccc}
\hline Image pair & Metric & Method1 & Method2 & Method3 & Proposed method \\
\hline MRI-PET & EI & 87.0194 & 87.0603 & 87.0303 & $\mathbf{8 7 . 0 7 3 1}$ \\
MRI-SPECT & EI & 68.9888 & 68.5563 & 68.4424 & $\mathbf{6 9 . 1 0 7 7}$ \\
\hline
\end{tabular}

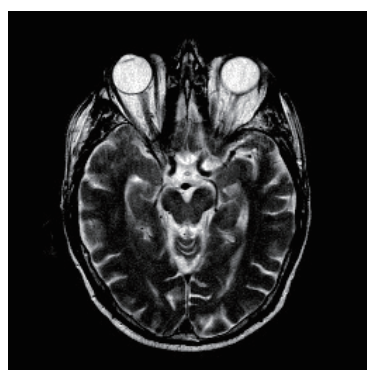

(a1)

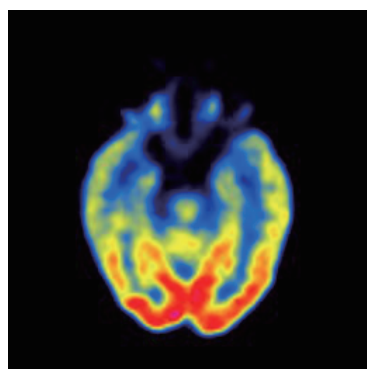

(b1)

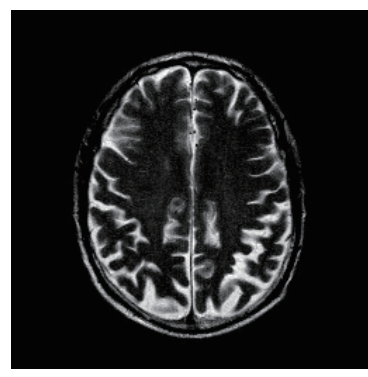

(a2)

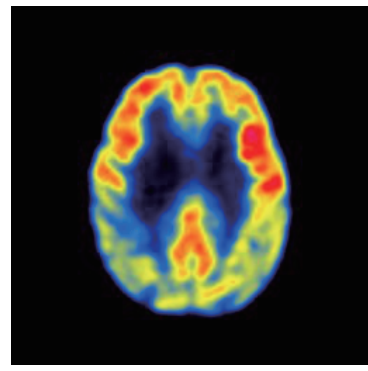

(b2)

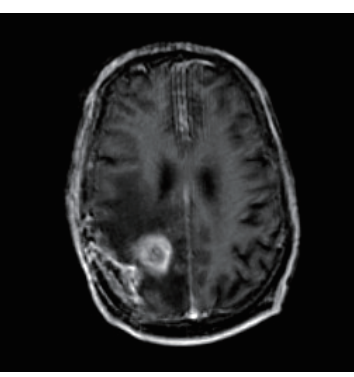

(a3)

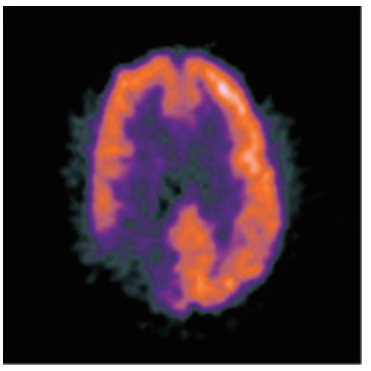

(b3)

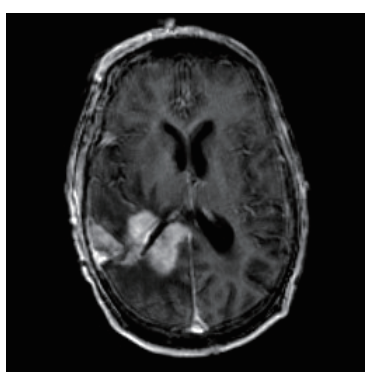

(a4)

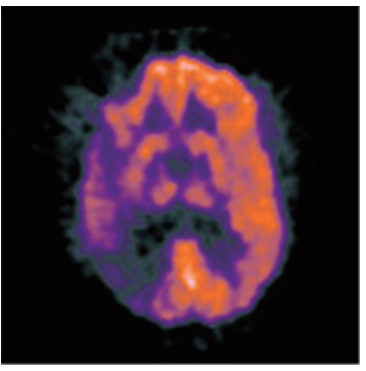

(b4)

图 7 (网络版彩图) 4 组 MRI-PET 实验图像. (a1) (a4) 是 MRI 图像, (b1) (b4) 是对应的 PET 图像

Figure 7 (Color online) Four MRI-PET image pairs. (a1) (a4) MRI images; (b1) (b4) corresponding PET images

其中 Method1 是指近似层使用局部能量取大, 细节层使用本文所提细节层融合方法. Method2 是 指近似层使用本文所提方法, 细节层使用输入图像的像素绝对值作为 PA-SPCNN 模型的外部刺激输 入的方法. Method 3 是指近似层和细节层分别使用局部能量取大和输入图像的像素绝对值作为 PASPCNN 模型的外部刺激输入的方法. 上述 3 种方法用于和本文所提算法做比较. 从表 2 中可以清晰 地看到, 本文所提算法具有最优的 EI 指标值, 即融合结果质量最好.

\section{3 主观分析}

为了更好地验证所提算法的有效性, 本文算法与以下方法做比较: LP-SR ${ }^{[35]}, \mathrm{NSCT}$-SF-PCNN ${ }^{[21]}$, $\mathrm{GFF}^{[12]}, \mathrm{LLF}-\mathrm{IOI}{ }^{[16]}, \mathrm{NSST}-\mathrm{PAPCNN}{ }^{[24]}$. 前 3 种方法是经典算法, 后两种方法是最新的医学图像融 合算法, 所有方法的参数设置与对应文献一致. 上述 5 种方法的实验源代码可在网址122)314)5) 上获取. 此外, 本文选取 40 组医学图像用于实验, 其中 20 组 MRI-PET 医学图像, 20 组 MRI-SPECT 医学图

1) The source code of the LP-SR method From Yu Liu's Website. http://www.escience.cn/people/liuyu1/Codes.html.

2) The source code of the NSCT-SF-PCNN method From Xiaobo Qu's Website. http://csrc.xmu.edu.cn/.

3) The source code of the GFF method From Xudong Kang's Website. http://xudongkang.weebly.com/index.html.

4) The source code of the LLF-IOI method From Jiao Du's Website. http://www.escience.cn/people/xiaomi/index.html.

5) The source code of the NSST-PAPCNN method From Yu Liu's Website. http://www.escience.cn/people/liuyu1/ Codes.html. 


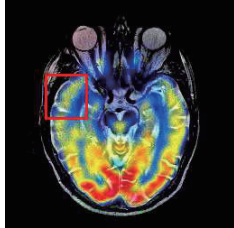

(a1)

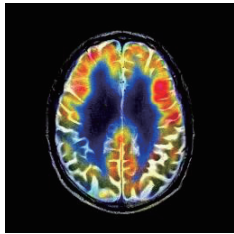

(b1)

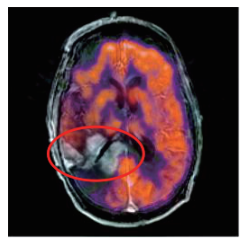

(c1)

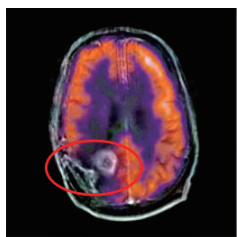

(d1)

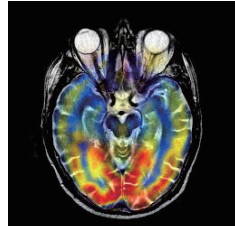

(a2)

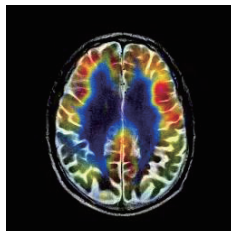

(b2)

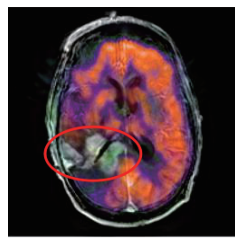

(c2)

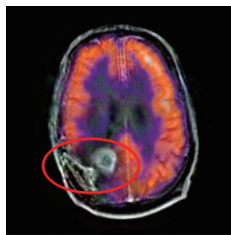

(d2)

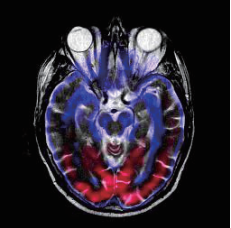

(a3)

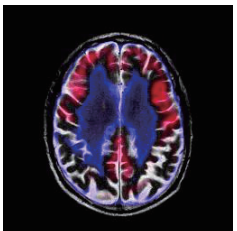

(b3)

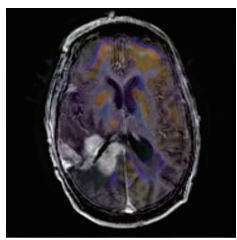

(c3)

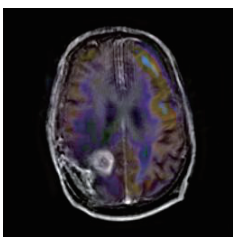

(d3)

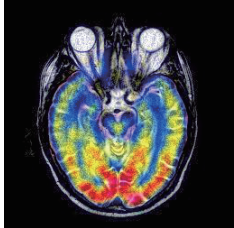

(a4)

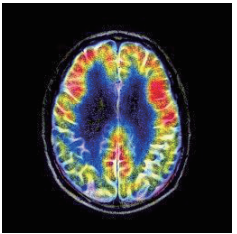

(b4)

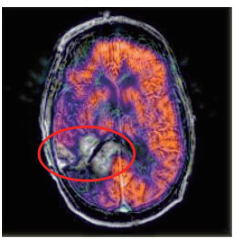

(c4)

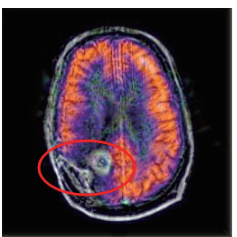

(d4)

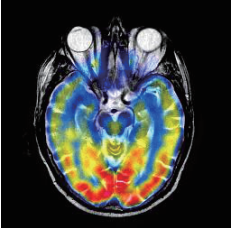

(a5)

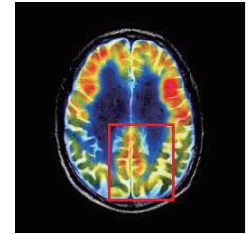

(b5)

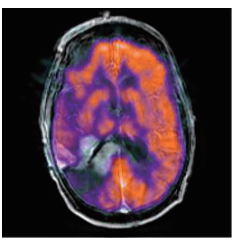

(c5)

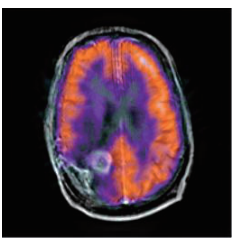

(d5)

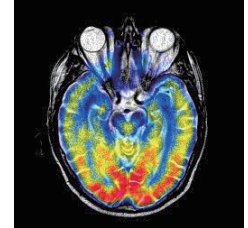

(a6)

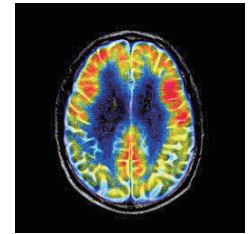

(b6)

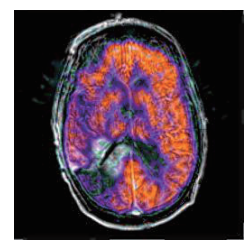

(c6)

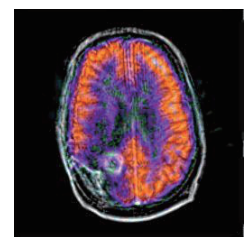

(d6)

图 8 (网络版彩图) 4 组 MRI-PET 实验图像用不同方法获得的融合结果对比图. (a) (d) 分别为 4 组实验结果, 每组从左到右依次为 LP-SR, NSCT-SF-PCNN, GFF, LLF-IOI, NSST-PAPCNN 和本文所提算法得到的 融合图像

Figure 8 (Color online) Comparison of fusion results obtained by different methods on 4 MRI-PET image pairs. (a) $\sim(d)$ are fusion results on the 4 pairs obtained by LP-SR, NSCT-SF-PCNN, GFF, LLF-IOI, NSST-PAPCNN, and the proposed method, respectively

像. 本文所有的实验图像都是来自于哈佛大学医学院的全脑图谱数据库 (The Whole Brain Atlas $)^{6)}$. 为 了更好地观察本文所提算法的视觉效果, 本文从 40 组实验数据里面选取 4 组 MRI-PET 医学图像和 4 组 MRI-SPECT 医学图像, 再分别使用上述 5 种文献方法与本文所提算法获得融合结果图像, 并对 比融合结果观察本文所提算法的有效性. 图 7 是选取的 4 组 MRI-PET 实验图像, 图 8 是不同方法作 用于此实验图像的融合结果对比图. 同理, 图 9 和 10 是选取的 MRI-SPECT 的实验图像和融合结果 对比图.

图 8 是选取了 20 组 MRI-PET 实验图像中的 4 组通过不同的方法获得的结果对比图, 每一行 表示同一组实验图像使用不同方法获得的融合结果, 其中前 3 幅是 3 种经典的图像融合算法 (依次 是 LP-SR, NSCT-SF-PCNN, GFF) 的融合结果, 第 4 和 5 幅是最新医学图像融合算法 (LLF-IOI 和 NSST-PAPCNN) 的融合结果, 最后一幅图是本文所提算法的融合结果. 由于本文探讨的是解剖图像和 功能图像的融合, 判断融合图像的优劣应该主要考虑两个方面信息, 一方面是解剖图像 (MRI 医学图 像) 提供的结构细节信息应该被尽可能地全部融合, 另一方面是功能图像 (PET 医学图像或 SPECT

6) Whole Brain Atlas. http://www.med.harvard.edu/AANLIB. 


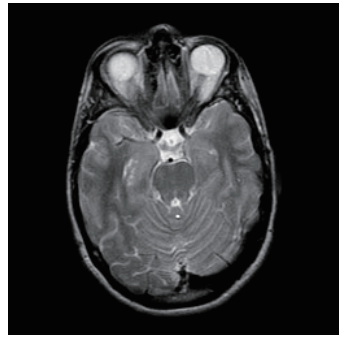

(a1)

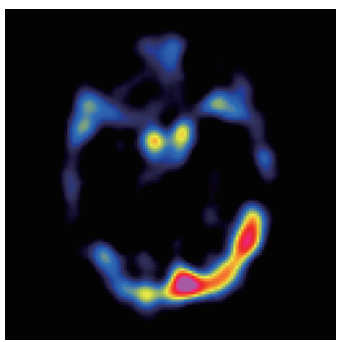

(b1)

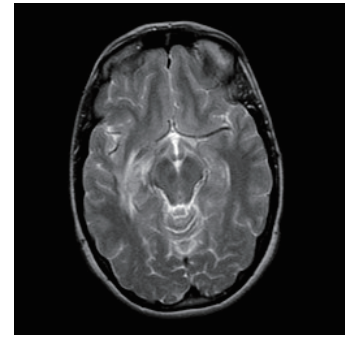

(a2)

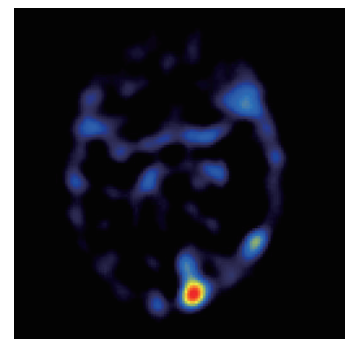

(b2)

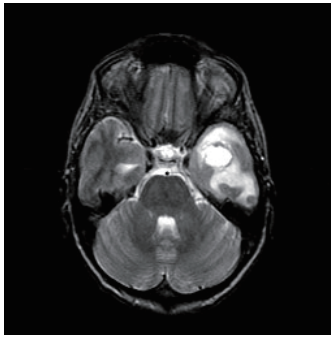

(a3)

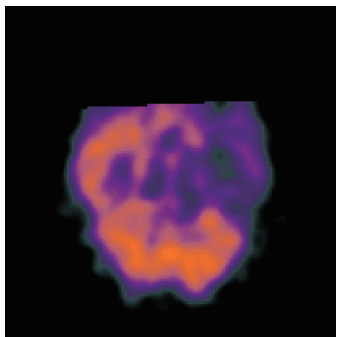

(b3)

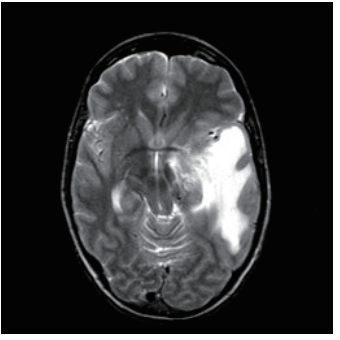

(a4)

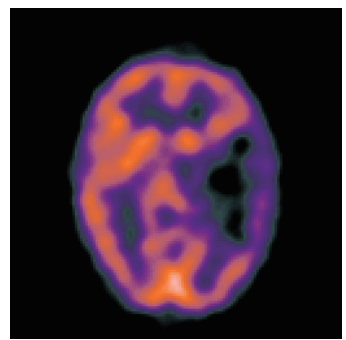

(b4)

图 9 (网络版彩图) 4 组 MRI-SPECT 实验图像. (a1) (a4) 是 MRI 图像, (b1) (b4) 是对应的 SPECT 图像

Figure 9 (Color online) Four MRI-SPECT image pairs. (a1) (a4) MRI images; (b1) (b4) corresponding SPECT images

医学图像) 提供的颜色信息应该尽可能地减少光谱扭曲或颜色失真. 通过比较原图像与结果对比图 (图 7 和 8), 可以观察到, 在结构信息的融合方面, 只有 LP-SR 方法融合结果 (图 8(a1), (b1), (c1), (d1) ) 的部分细节呈现较少, 或被颜色信息覆盖细节不清楚, 红色方框标注的区域明显地显示这一缺 陷. 此外, 每组实验图像的不同方法结果之间最大的差异在于颜色信息的保留, 观察所有结果, 不难 看出只有 NSST-PAPCNN 方法和本文所提算法的融合结果 (图 8(a5), (a6), (b5), (b6), (c5), (c6), (d5), (d6)) 展示出最优的颜色保留能力. 三类经典方法的融合结果 (图 8 每一行的前 3 幅) 存在或 多或少的颜色失真, 特别是 GFF 融合结果存在严重的颜色失真现象, 严重影响融合图像的下一步应 用. LLF-IOI 方法尽管有明显的细节增强效果 (见图 8(a4), (b4), (c4), (d4)), 但是多处地方明显出 现了颜色失真, 用红色椭圆框标注的区域便是上述几处明显的颜色失真区域. NSST-PAPCNN 方法 有很好的实验结果 (见图 8(a5), (b5), (c5), (d5)), 相对较好地融合了结构信息与颜色信息, 但是它的 结果过于光滑, 出现了一些不利于观察 MRI 图像的结构信息; 再者, 相比于本文所提算法的融合结 果, 该方法结果的部分区域有轻微的颜色失真, 如图 8(b5) 红色方框所标注区域. 与其他方法相比, 本文所提算法不仅使光谱信息和结构信息都十分恰当地融合到了结果图像中, 还在融合过程中适当 地增强了融合图像的细节信息, 使得融合结果信息更丰富, 更有利于图像的观察和它在临床方面的 应用。

从 MRI-PET 医学图像的融合过程中可知, 解剖图像的结构信息在加上功能图像的颜色信息后可 能会导致有的区域被颜色信息覆盖, 不利于观察解剖图像的细节. 由于本文使用了可以增加细节的分 解和重构工具, 从而实现了融合图像中解剖图像的细节在加上颜色信息后依旧清晰的效果. 这一优势 在 MRI-SPECT 图像的融合中可非常清晰地观察到. 图 9 是本文选取的 4 组 MRI-SPECT 实验图 像, 图 10 是使用不同的融合方法得到的融合结果. 从图 10 中可得, 只有 LP-SR 和 NSCT-SF-PCNN 两种方法结果的 MRI 结构信息明显不足, 其他方法都能较好地将结构信息融合到结果图像中. 颜色 


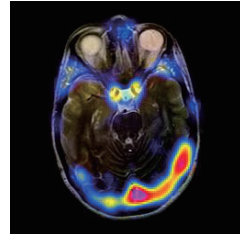

(a1)

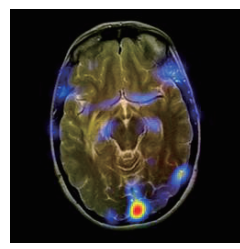

(b1)

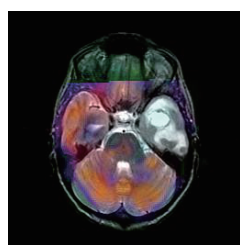

(c1)

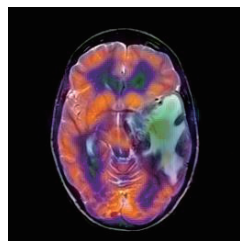

(d1)

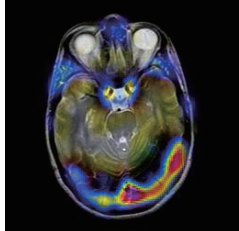

(a2)

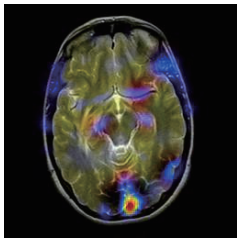

(b2)

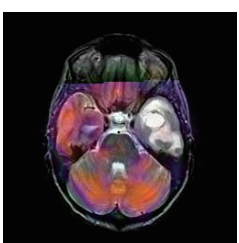

(c2)

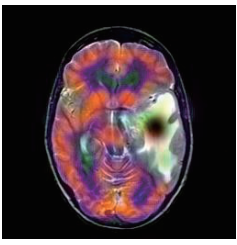

(d2)

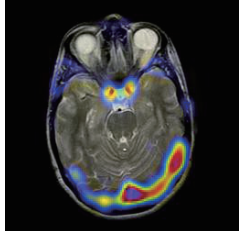

(a3)

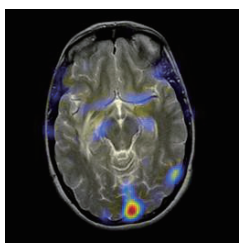

(b3)

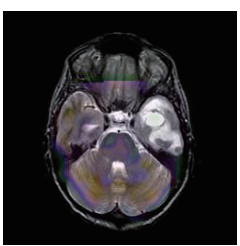

(c3)

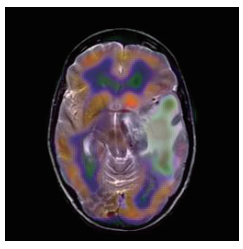

(d3)

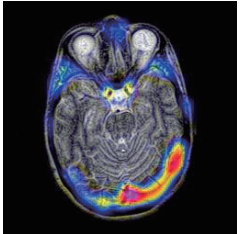

(a4)

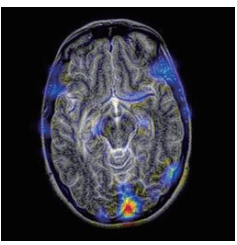

(b4)

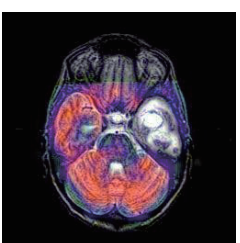

(c4)

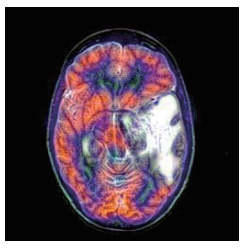

(d4)

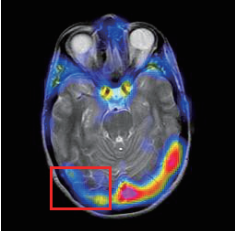

(a5)

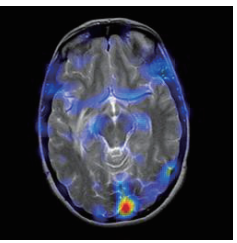

(b5)

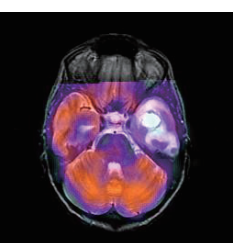

(c5)

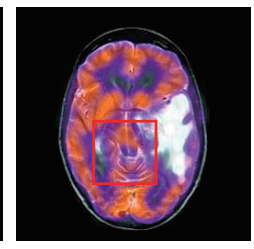

(d5)

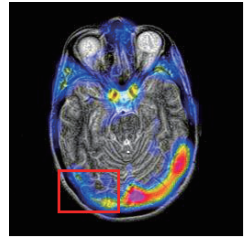

(a6)

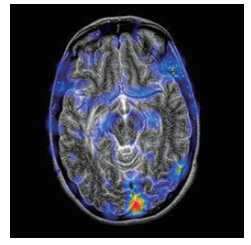

(b6)

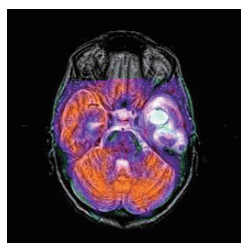

(c6)

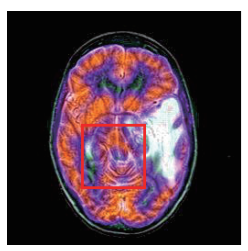

(d6)

图 10 (网络版彩图) 4 组 MRI-SPECT 实验图像用不同方法获得的融合结果对比图. (a) (d) 分别为 4 组实验 结果, 每组从左到右依次为 LP-SR, NSCT-SF-PCNN, GFF, LLF-IOI, NSST-PAPCNN 和本文所提算法 得到的融合图像

Figure 10 (Color online) Comparison of fusion results obtained by different methods on 4 MRI-SPECT image pairs. (a) (d) are fusion results on the 4 pairs obtained by LP-SR, NSCT-SF-PCNN, GFF, LLF-IOI, NSST-PAPCNN, and the proposed method, respectively

信息的保留程度直接关系着融合图像的质量, 观察图 10 可知, 图 10(a1) (a4), (b1) (b4), (c1) (c4), $(\mathrm{d} 1) \sim(\mathrm{d} 4)$ 存在不同程度的颜色失真, 这都严重影响了结果图像的视觉效果. 从图 10 中可以发现视觉 效果最好的是每组最后两幅融合结果图像, 它们分别是 NSST-PAPCNN 方法和本文所提算法的融合 结果. 比较图 10(a5) 和 (a6), (b5) 和 (b6), (c5) 和 (c6), (d5) 和 (d6), 可以注意到在 NSST-PAPCNN 方 法的融合结果图像中, SPECT 的颜色信息覆盖了 MRI 的细节信息, 使得 MRI 的细节在融合图像中基 本观察不到 (见图 10(b5) 和 (c5) 红色方框区域), 而本文所提算法的融合结果在该处却可以清楚观察 到 MRI 的细节信息, 同时保留 SPECT 的颜色信息 (见图 10(b6) 和 (c6) 红色方框区域). 这主要是因 为本文所提算法有细节增强的效果, 在融合图像中, 不仅保留了颜色信息还增强了 MRI 的结构信息, 使得二者在信息融合的同时都可以被观察到并被加以利用. 此外, 众所周知, 医学图像的细节在诊断 过程中至关重要, 如若融合过程中可以将不太明显的细节增强, 将有助于医生更清晰地观察细微病变, 尽快为病人制定合理有效的治疗方案、缩短治疗周期.

\section{4 客观评价}

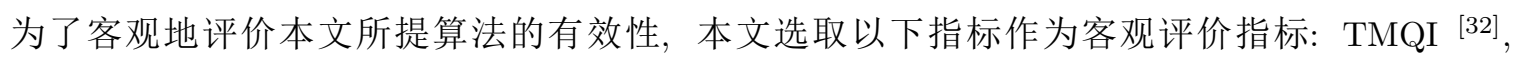


$\mathrm{SF}^{[33]}, \mathrm{EI}^{[34]}$ 和互信息 $(\mathrm{MI})^{[36]}$.

\subsection{1 客观评价指标}

TMQI ${ }^{[32]}$ 度量参考图像和融合图像之间的亮度与对比度显著特征, 测量融合图像的结构保真度, 指标值越大代表融合图像有更高的空间分辨率, 从输入图像中获得更多的亮度信息, 融合图像信息越 全面.

$$
\operatorname{TMQI}(I, F)=a T^{\alpha}+(1-a) H^{\beta},
$$

其中 $T$ 表示参考图像与融合图像的结构保真度, $H$ 表示融合图像的统计自然度, 其余参数默认为 $a=0.8012, \alpha=0.3046, \beta=0.7088$. TMQI 的值是 $\operatorname{TMQI}(F, A)$ 和 $\operatorname{TMQI}(F, B)$ 的平均.

$\mathrm{SF}^{[33]}$ 描述图像在空间域的梯度分布, 度量图像的细节丰富程度, 值越大代表图像细节越清晰, 计 算公式如下:

$$
\mathrm{SF}=\sqrt{(\mathrm{RF})^{2}+(\mathrm{CF})^{2}}
$$

其中,

$$
\mathrm{RF}=\sqrt{\frac{1}{M N} \sum_{x=1}^{M} \sum_{y=1}^{N}[F(x, y)-F(x, y-1)]^{2}}, \quad \mathrm{CF}=\sqrt{\frac{1}{M N} \sum_{x=1}^{M} \sum_{y=1}^{N}[F(x, y)-F(x-1, y)]^{2}},
$$

$F$ 表示融合图像, $(x, y)$ 表示像素位置, $(M, N)$ 表示图像像素矩阵大小.

$\mathrm{EI}^{[34]}$ 值越大代表图像边缘越锐化, 融合图像质量越好, 计算公式如下:

$$
\mathrm{EI}=\frac{1}{M N} \sqrt{\left(G_{x}\right)^{2}+\left(G_{y}\right)^{2}},
$$

其中 $G_{x}$ 和 $G_{y}$ 是 Sobel 边缘检测算子通过卷积模板 $w$ 和 $w^{\prime}$ 分别与融合图像 $F$ 做平面卷积得到的, 其中

$$
w=\left[\begin{array}{ccc}
1 & 2 & 1 \\
0 & 0 & 0 \\
-1 & -2 & -1
\end{array}\right], \quad w^{\prime}=\left[\begin{array}{ccc}
1 & 0 & -1 \\
2 & 0 & -2 \\
1 & -0 & -1
\end{array}\right] .
$$

MI ${ }^{[36]}$ 衡量融合图像中来源于原图像的信息比例, 计算公式如下:

$$
\operatorname{MI}(F, I)=\sum_{x, y} P_{F I}(x, y) \log \frac{P_{F I}(x, y)}{P_{F}(x) \cdot P_{I}(y)},
$$

其中 $P_{F I}(x, y)$ 是联合概率分布, $P_{F}(x)$ 和 $P_{I}(y)$ 是边缘概率分布. $F$ 表示融合图像, $I$ 是输入原图像, 最终的 $\mathrm{MI}$ 值是 $\operatorname{MI}(F, A)$ 和 $\mathrm{MI}(F, B)$ 的平均值.

\subsection{2 客观评价}

表 3 是 20 组 MRI-PET 实验图像经过 6 种方法获得的融合结果的客观评价指标平均值, 并给出 了本文所提算法的各个指标中的排名, 表中黑体加粗表示最优值. 从表 3 中可看出, 本文所提算法的 $\mathrm{SF}, \mathrm{EI}$ 和 TMQI 等 3 个客观指标平均值是最优的. 这是因为本文所提算法有细节增强的效果, 使得所 有实验结果图像有更清晰的梯度与边缘, 更丰富的细节信息, 而前两个指标最能刻画本文所提算法在 此方面的优越性. 除了细节保留与增强的优势, 也唯有本文所提算法的颜色保真度最高, 故 TMQI 指 
表 320 组 MRI-PET 图像融合结果的客观评价指标平均值

Table 3 Average evaluation values of fusion results on 20 MRI-PET image pairs

\begin{tabular}{ccccccc}
\hline Metric & LP-SR & NSCT-SF-PCNN & GFF & LLF-IOI & NSST-PAPCNN & Proposed method \\
\hline SF & 23.789 & 24.992 & 24.709 & 27.731 & 24.219 & $\mathbf{2 9 . 5 2 5 ( 1 )}$ \\
EI & 68.256 & 73.319 & 72.408 & 82.738 & 71.263 & $\mathbf{8 6 . 1 3 1 ( 1 )}$ \\
MI & 5.017 & 4.957 & $\mathbf{5 . 1 6 8}$ & 4.997 & 5.109 & $5.076(3)$ \\
TMQI & 0.732 & 0.719 & 0.683 & 0.734 & 0.735 & $\mathbf{0 . 7 3 7}(1)$ \\
\hline
\end{tabular}

表 420 组 MRI-SPECT 图像融合结果的客观评价指标平均值

Table 4 Average evaluation values of fusion results on 20 MRI-SPECT image pairs

\begin{tabular}{ccccccc}
\hline Metric & LP-SR & NSCT-SF-PCNN & GFF & LLF-IOI & NSST-PAPCNN & Proposed method \\
\hline SF & 17.350 & 18.075 & 16.866 & 22.320 & 17.424 & $\mathbf{2 3 . 4 6 4 ( 1 )}$ \\
EI & 49.368 & 51.370 & 48.281 & 67.992 & 50.481 & $\mathbf{7 2 . 2 0 7 ( 1 )}$ \\
MI & 4.466 & 4.459 & 4.723 & 4.618 & $\mathbf{4 . 7 6 8}$ & $4.676(3)$ \\
TMQI & 0.730 & 0.715 & 0.719 & $\mathbf{0 . 7 3 0}$ & 0.726 & $0.729(2)$ \\
\hline
\end{tabular}

标也是最优的值. 此外, 本文所提算法的 MI 指标排在第 3 位, 前两位是 GFF 和 NSST-PAPCNN 方 法, 这个问题产生的根本原因在于本文所提算法的融合图像在融合过程被有意增强细节以便更好地应 用于临床诊断, 以提高医生观察疾病的准确率. 但是, 观察前面的实验结果对比图可知, GFF 方法的颜 色失真较严重, 与原功能图像的颜色大不相同; 而 NSST-PAPCNN 方法与本文所提方法相比, 融合结 果图像也有些轻微的颜色失真. 尽管本文所提算法的个别指标数据结果不是最优的, 但是综合对每幅 结果图像的仔细观察可知, 本文算法获得的结果图像质量最优.

表 4 是 20 组 MRI-SPECT 实验图像经过 6 种方法获得的融合结果的客观评价指标平均值, 同样 给出了本文所提算法在各个指标上的排名, 表中加粗字表示最优值. 从表 4 中可以看出, 本文所提方 法在 SF 和 $\mathrm{EI}$ 指标上都是最优的, MI 指标排在第 3 位, 这些指标的值与表 3 的指标排名一致, 都是 因为本文所提算法在融合过程中有细节增强的过程, 增强了图像的对比度、清晰度, 故 SF 和 EI 指标 值是最优的，同时计算互信息时又受制于增强的细节信息，导致互信息的指标值不是最高. 本文所提 算法的 TMQI 指标排在第 2 位, 但是与并列排名第一的 LP-SR 和 LLF-IOI 方法相比只相差 0.001 , 这 说明本文算法具有较好的结构保真度; 结合 4.3 小节可知, LP-SR 方法的结果图像在结构信息和颜色 信息方面都没有本文所提算法好. 此外, 由于 LLF-IOI 方法没有进行颜色空间转换直接在原像素上进 行融合操作, 以致于结果图像的整体颜色有些暗沉, 影响视觉效果. 总体上讲, 上述两种方法的融合结 果图像的视觉效果明显差于本文所提算法.

图 11 是 40 组解剖图像与功能图像使用不同方法获得的融合图像的客观评价指标平均值的柱状 图, 用来综合评价本文所提算法与其他方法的优劣. 从图中可观察到, 本文所提算法的指标平均值在 SF, EI, TMQI 3 个指标上是最优的, 这几个指标代表着本文所提算法有更清晰的细节, 更锐化的边缘, 并且拥有最优的保真性能. 此外, 本文所提算法只在 MI 指标上略逊于 GFF 方法和 NSST-PAPCNN 方法, 而这与本文所提算法有意增强细节有关.

\subsection{3 算法复杂度分析}

表 5 是本文所提到的 6 种方法用于医学图像融合的时间比较, 从表中可以看到, 本文所提算法用 

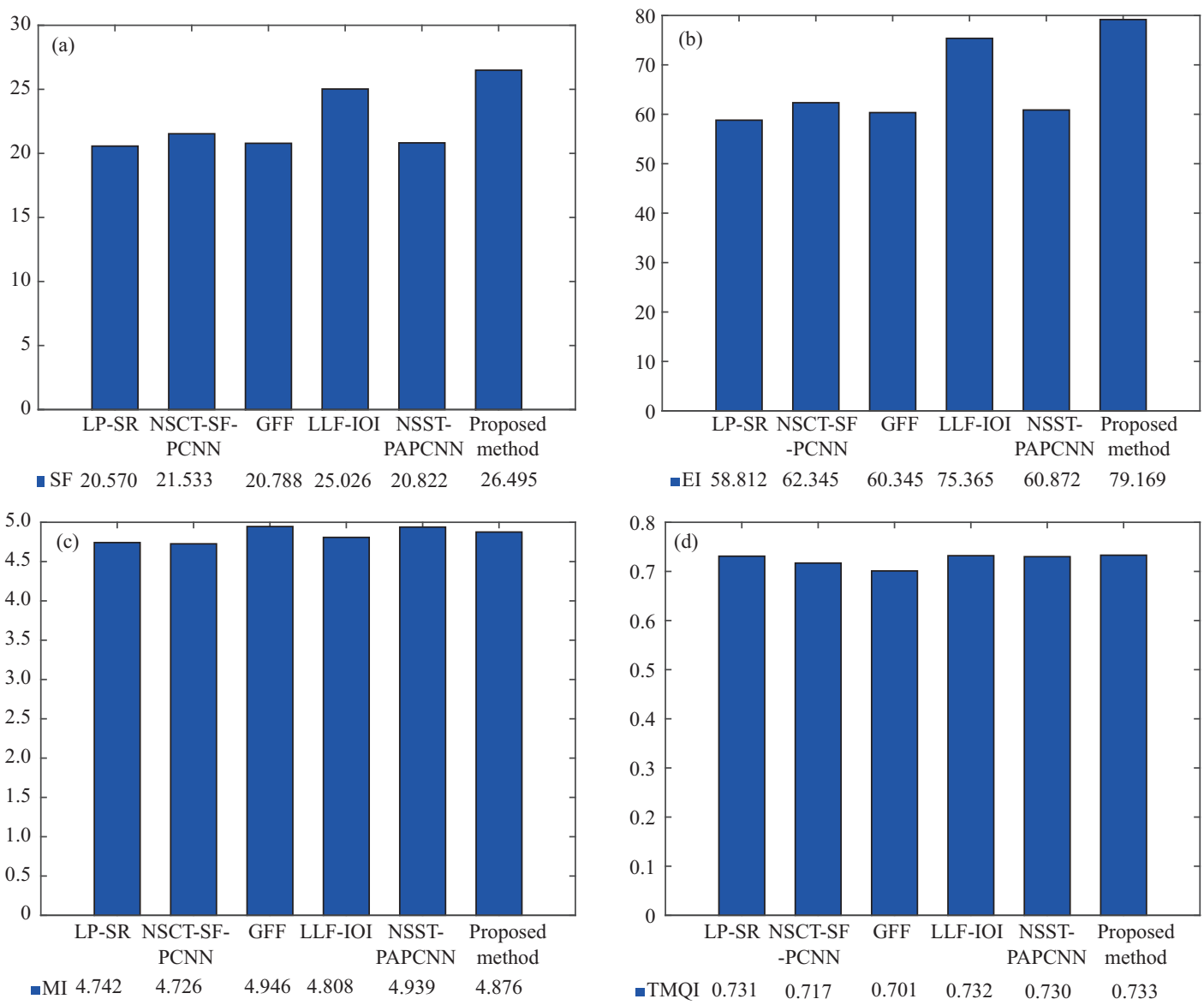

图 11 (网络版彩图) 40 组实验图像使用不同方法获得的融合图像的客观指标平均值的柱状图

Figure 11 (Color online) Histograms of the average evaluation values of fusion results obtained by different methods on 40 experimental image pairs. (a) SF; (b) EI; (c) MI; (d) TMQI

表 5 不同融合算法的运行时间

Table 5 Running time of different fusion methods

\begin{tabular}{ccccccc}
\hline Metric & LP-SR & NSCT-SF-PCNN & GFF & LLF-IOI & NSST-PAPCNN & Proposed method \\
\hline Time & 0.046056 & 260.860688 & 0.246661 & 275.129536 & 15.992219 & 331.249379 \\
\hline
\end{tabular}

的时间最长, 这主要是因为 CSF 和 LLF 两个方法在运行过程中都比较耗时, 但是基于本文的出发点, CSF 可以是根据颜色刺激得到显著性值, 这一步提取彩色显著特征的操作充分地考虑了颜色亮度信息 的视觉效果, 而 LLF 用于图像分解与重构可以将细节增强, 使得解剖图像的结构信息和功能图像的颜 色信息能够同时在各类图像中被很好地呈现出来, 故为了得到好的融合结果并使其具有更高的质量和 更广泛的应用价值, 时间代价是值得付出的.

综上分析, 尽管本文所提算法在时间上优势不显著, 但是, 与经典的融合规则和现存的医学图像融 合算法相比, 本文所提医学图像融合算法在客观指标和主观评价方面都有一定的优势. 


\section{5 总结}

本文针对多模态医学图像不同的成像特点, 同时为避免功能图像的颜色信息弱化解剖图像的细节 信息, 选取了可以增强细节的局部拉普拉斯滤波作为融合过程的分解和重构工具, 使得图像细节在分 解和重构过程中能够增强, 且融合图像中的结构信息和颜色信息都能很好地被观察到. 鉴于近似图主 要包含原图像的能量信息和大致轮廓, 本文结合局部能量和梯度域引导滤波先引出边缘能量的概念及 其计算方法, 再提出一个改进的局部能量取大融合规则用于近似图融合. 此外, 改进的拉普拉斯和与 彩色显著特征分别作为解剖图像和功能图像在参数自适应简化脉冲神经网络模型中的外部刺激输入, 这一选取充分地考虑了解剖图像的结构信息和功能图像的颜色显著信息的不同呈现方式. 最后, 通过 仿真实验表明本文所提融合规则的可行性和医学图像融合算法的有效性. 相比于经典的融合算法和最 新的医学图像融合算法, 本文所提算法在主观和客观方面都有一定优势.

\section{参考文献}

1 James A P, Dasarathy B V. Medical image fusion: a survey of the state of the art. Inf Fusion, 2014, 19: 4-19

2 Yang Y, Han C, Kang X, et al. A novel image fusion algorithm based on IHS and discrete wavelet transform. In: Proceedings of the IEEE International Conference on Automation \& Logistics, Jinan, 2007. 1936-1940

3 Selesnick I W, Baraniuk R G, Kingsbury N C. The dual-tree complex wavelet transform. IEEE Signal Process Mag, 2005, 22: 123-151

4 Burt P J, Adelson E H. The Laplacian pyramid as a compact image code. Readings Comput Vision, 1987, 31: 671-679

5 Toet A. Hierarchical image fusion. Machine Vis Appl, 1990, 3: 1-11

6 Cunha A L D, Zhou J, Do M N. The nonsubsampled contourlet transform: theory, design, and applications. IEEE Trans Image Process, 2006, 15: 3089-3101

7 Easley G, Labate D, Lim W Q. Sparse directional image representations using the discrete shearlet transform. Appl Comput Harmonic Anal, 2008, 25: 25-46

8 Liu X B, Mei W B, Du H Q. Detail-enhanced multimodality medical image fusion based on gradient minimization smoothing filter and shearing filter. Med Biol Eng Comput, 2018, 56: 1565-1578

9 Liu X, Mei W, Du H. Multimodality medical image fusion algorithm based on gradient minimization smoothing filter and pulse coupled neural network. Biomed Signal Process Control, 2016, 30: 140-148

10 Zhang Q, Shen X, Xu L, et al. Rolling guidance filter. In: Proceedings of the European Conference on Computer Vision, Zurich, 2014. 815-830

11 Paris S, Hasinoff S W, Kautz J. Local Laplacian filters. ACM Trans Graph, 2011, 30: 1244-1259

12 Li S T, Kang X D, Hu J W. Image fusion with guided filtering. IEEE Trans Image Process, 2013, 22: 2864-2875

13 Liu X, Mei W, Du H. Structure tensor and nonsubsampled shearlet transform based algorithm for CT and MRI image fusion. Neurocomputing, 2017, 235: 131-139

14 Du J, Li W, Xiao B, et al. Union Laplacian pyramid with multiple features for medical image fusion. Neurocomputing, 2016, 194: 326-339

15 Du J, Li W, Xiao B, et al. Medical image fusion by combining parallel features on multi-scale local extrema scheme. Knowledge-Based Syst, 2016, 113: 4-12

$16 \mathrm{Du}$ J, Li W, Xiao B. Anatomical-functional image fusion by information of interest in local Laplacian filtering domain. IEEE Trans Image Process, 2017, 12: 5855-5866

17 Wu Z, Huang Y, Zhang K. Remote sensing image fusion method based on PCA and curvelet transform. J Ind Soc Remote Sens, 2018, 46: 687-695

18 Zhang K, Huang Y, Zhao C. Remote sensing image fusion via RPCA and adaptive PCNN in NSST domain. Int J Wavelets Multiresolut Inf Process, 2018, 16: 1850037

19 Zhu Z Q, Yin H, Chai Y, et al. A novel multi-modality image fusion method based on image decomposition and sparse representation. Inf Sci, 2018, 432: 516-529

20 Aishwarya N, Thangammal C B. A novel multimodal medical image fusion using sparse representation and modified 
spatial frequency. Int J Imag Syst Technol, 2018, 28: 175-185

21 Qu X B, Yan J W, Xiao H Z, et al. Image fusion algorithm based on spatial frequency-motivated pulse coupled neural networks in nonsubsampled contourlet transform domain. Acta Autom Sin, 2008, 34: 1508-1514

22 Xiong Y, Wu Y, Wang Y, et al. A medical image fusion method based on SIST and adaptive PCNN. In: Proceedings of the Control \& Decision Conference, Chongqing, 2017. 5189-5194

23 Zhan K, Zhang H J, Ma Y D. New spiking cortical model for invariant texture retrieval and image processing. IEEE Trans Neural Netw, 2009, 20: 1980-1986

24 Yin M, Liu X, Liu Y, et al. Medical image fusion with parameter-adaptive pulse coupled neural network in nonsubsampled shearlet transform domain. IEEE Trans Instrum Meas, 2019, 68: 49-64

25 Yin M, Duan P, Liu W, et al. A novel infrared and visible image fusion algorithm based on shift-invariant dual-tree complex shearlet transform and sparse representation. Neurocomputing, 2017, 226: 182-191

26 Goferman S, Zelnik-Manor L, Tal A. Context-aware saliency detection. IEEE Trans Pattern Anal Mach Intell, 2012, 34: 1915-1926

27 Kou F, Chen W, Wen C, et al. Gradient domain guided image filtering. IEEE Trans Image Process, 2015, 24: $4528-4539$

28 He K, Sun J, Tang X. Guided image filtering. IEEE Trans Pattern Anal Mach Intell, 2013, 35: 1397-1409

29 Chen Y L, Park S K, Ma Y D, et al. A new automatic parameter setting method of a simplified PCNN for image segmentation. IEEE Trans Neural Netw, 2011, 22: 880-892

30 Yang Y, Que Y, Huang S, et al. Multimodal sensor medical image fusion based on type-2 fuzzy logic in NSCT domain. IEEE Senss J, 2016, 16: 3735-3745

31 Liu Y, Chen X, Cheng J, et al. A medical image fusion method based on convolutional neural networks. In: Proceedings of the International Conference on Information Fusion, Xi'an, 2017. 1070-1076

32 Yeganeh H, Wang Z. Objective quality assessment of tone-mapped images. IEEE Trans Image Process, 2013, 22: 657-667

33 Eskicioglu A M, Fisher P S. Image quality measures and their performance. IEEE Trans Commun, 1995, 43: 2959-2965

34 Wang Y, Du H, Xu J, et al. A no-reference perceptual blur metric based on complex edge analysis. In: Proceedings of the Network Infrastructure and Digital Content (IC-NIDC), Beijing, 2012. 487-491

35 Liu Y, Liu S, Wang Z. A general framework for image fusion based on multi-scale transform and sparse representation. Inf Fusion, 2015, 24: 147-164

36 Qu G, Zhang D, Yan P. Information measure for performance of image fusion. Electron Lett, 2002, 38: 313-315 


\title{
Medical-image-fusion algorithm based on a detail-enhanced and pulse-coupled neural-network model stimulated by parallel fea- tures
}

\author{
Guofen WANG ${ }^{1} \&$ Yongdong HUANG ${ }^{1,2^{*}}$ \\ 1. Institute of Image Processing and Understanding, North Minzu University, Yinchuan 750021, China; \\ 2. School of Science, Dalian Minzu University, Dalian 116600, China \\ * Corresponding author. E-mail: huang_yongdong@163.com
}

\begin{abstract}
The purpose of medical-image fusion is to integrate the comprehensive information of multimodal medical images into a single image, which is helpful for clinical diagnosis, increasing the accuracy of disease observation by physicians, and shortening the treatment period. A new fusion algorithm for anatomical and functional images is proposed in this paper. Local Laplacian filtering (LLF) is chosen as the decomposition tool in the fusion process, which can enhance the details, protect the edges, and ensure that the details of the anatomical features in the fused images cannot be covered by the color information of the functional images. The steps of this algorithm are as follows: firstly, LLF decomposes the original image into an approximate image and a series of detailed images. Secondly, for the approximate image, this paper proposes a fusion rule for an improved local-energy maximum by combining the regional and edge energies. For detailed images, the parameter-adaptive simplified-pulse-coupled neural-network (PA-SPCNN) model is used to fuse the detailed images. The novel summodified-Laplacian and color-saliency feature are selected as external-stimulus inputs of the PA-SPCNN model in the anatomical and functional images, respectively. Finally, the fusion image is obtained by an inverse-LLF transform. Simulation experiments show that the proposed algorithm is superior to the classical algorithm in subjective and objective evaluation.
\end{abstract}

Keywords image processing, image fusion, medical image processing, local Laplacian filtering, pulse-coupledneural-network, novel sum-modified-Laplacian, color saliency feature, edge energy

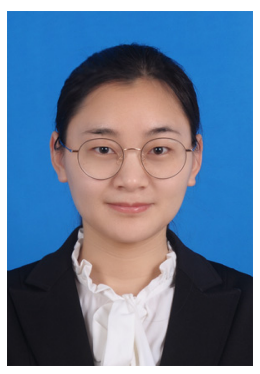

Guofen WANG was born in 1995. She received her bachelor's degree in mathematics from North Minzu University, Yinchuan, in 2016. Currently, she is enrolled as a master's candidate in applied mathematics. Her research interest is medical-image processing.

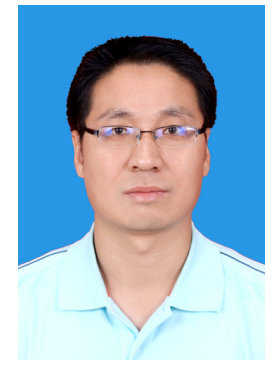

Yongdong HUANG was born in 1974 He received his Ph.D. in computational mathematics from Xi'an Jiaotong University, Xi'an, in 2007. He received his B.S and M.S degrees in mathematics from Ningxia University, in 1996 and 1999, respectively. Currently, he is a professor at North Minzu University and Dalian Minzu University. His research interests include wavelet analysis and information and image processing. 